\title{
The scaling of spatial attention in visual search and its modification in healthy aging
}

\author{
P. M. GREENWOOD and RAJA PARASURAMAN \\ Catholic University of America, Washington, D.C.
}

\begin{abstract}
A model of visual search (Greenwood \& Parasuraman, 1999) postulating that visuospatial attention is composed of two processing components-shifting and scaling of a variable-gradient attentional focus-was tested in three experiments. Whereas young participants are able to dynamically constrict or expand the focus of visuospatial attention on the basis of prior information, in healthy aging individuals visuospatial attention becomes a poorly focused beam, unable to be constricted around one array element. In the present work, we sought to examine predictions of this view in healthy young and older participants. An attentional focus constricted in response to an element-sized precue had the strongest facilitatory effect on visual search. However, this was true only when the precue correctly indicated the location of a target fixed in size. When precues incorrectly indicated target location or when target size varied, the optimal spatial scale of attention for search was larger, encompassing a number of array elements. Healthy aging altered the deployment of attentional scaling: The benefit of valid precues on search initially (in participants 65-74 years of age) was increased but later (in those 75-85 years of age) was reduced. The results also provided evidence that cue size effects are attentional, not strategic. This evidence is consistent with the proposed model of attentional scaling in visual search.
\end{abstract}

Searching for objects of interest in visual space, an important aspect of daily life, is often guided by prior knowledge about the objects. For example, the ability to predict whether the movement of leaves in a tree is caused by a bird or a squirrel requires deducing the likely identity of the animal obscured within the leaves by observing the size of the disturbed region in the tree. A small region of disturbance would signal the likely presence of a bird, whereas a larger region would indicate a squirrel. In this way, prior knowledge of target size and location can facilitate perception of a target (Eriksen \& St. James, 1986; Greenwood \& Parasuraman, 1999; Hawkins et al., 1990). The dominant model of visuospatial attention, Treisman's feature integration theory (FIT), claims that this process operates serially to bind features into an object (Treisman, 1996; Treisman \& Sato, 1990). However, the separate component processes of visuospatial attention, which are important for such target identification in visual search, are incompletely understood.

We have advanced a three-component model of visual search that attempts to characterize the way processing components of visuospatial attention are deployed in order to detect a target of interest embedded in distractors (Greenwood \& Parasuraman, 1999). We have argued that visual search depends on component processes of visuospatial

Correspondence concerning this article should be addressed to P. M. Greenwood, Cognitive Science Laboratory, Catholic University of America, Washington, DC 20064 (e-mail: greenwood@cua.edu).

Note-This article was accepted by the previous editorial team, headed by Neil Macmillan. attention - the malleable, movable resource that can heighten processing within regions of space where it is directed (Cave \& Bichot, 1999; Hawkins et al., 1990). These processes can be deployed as tools in visual search, depending on task demands. Important among these are (1) saccadic eye movements, (2) shifts in position of the attentional focus, and (3) dynamic adjustments of the scale of the attentional focus. Although processing components of visuospatial attention have been studied most often in relatively empty space (Castiello \& Umiltà, 1990; Posner, 1980), there is evidence that these processes are deployed differently in the presence of distractors (Gilchrist, Heywood, \& Findlay, 1999; Motter, 1993), inherent to the concept of visual search.

Much previous work on visuospatial attention has been concerned with the ability to change the location of the attentional focus - termed shifting. Indeed, it is this property of shifting the attentional focus that established theories of visual search seek to explain. FIT (Treisman \& Gormican, 1988) hypothesizes two stages in search. In the guided search (GS) model, which is based on FIT (Cave \& Wolfe, 1990), the parallel stage guides the serial stage in the shifting of visuospatial attention to the target. In addition to shifting, we have posited another characteristic of the attentional focus: the ability to adjust its size and densitytermed scaling. We have argued that the focus of visuospatial attention is a flexibly deployed gradient that can be dynamically constricted or expanded (Eriksen \& St. James, 1986) according to task demands (Greenwood \& Parasuraman, 1999). A constricted attentional focus, like a spotlight, could be adopted under conditions in which arrays are large and discrimination difficult, due to similar- 
ity between target and distractors. In contrast, under less demanding search conditions, such as those in which there are few distractors present or in which discrimination is easy, as in the phenomenon of popout, the optimal attentional focus would be a broader gradient. This enlarged and graded attentional focus may be better able to detect an easily discriminable target without the need for a saccade. An enlarged focus is also optimal when precues to target location are invalid - that is, located away from the target (Greenwood \& Parasuraman, 1999; Greenwood, Parasuraman, \& Alexander, 1997). Perhaps when the target appears at a distance, its properties can best be detected by a larger, more diffuse attentional focus. This view that visuospatial attention can be scaled flexibly reconciles the two dominant views of how visuospatial attention is deployed: a gradient (LaBerge, Brown, Carter, Bash, \& Hartley, 1991) or a spotlight (Posner, 1980). Thus, the gradient that is characteristic of the attentional focus would be constricted to resemble a spotlight when arrays are large and discrimination difficult.

This model also makes predictions about the effect of healthy aging on the deployment of visuospatial attention. The model claims that, in aging individuals, the attentional focus becomes a poorly focused beam, being both broader and less concentrated than that in young individuals. Furthermore, use of a poorly focused beam to locate targets increases dependence of the aging brain on such prior information as size and location cues. This heightened dependence is manifested as a greater effect of cue size on search speed in the young-old, as compared with young, individuals (Greenwood \& Parasuraman, 1999; Greenwood et al., 1997).

We have argued that visual search proceeds by deploying the two processing components of visuospatial attention to guide the third component, saccadic eye movements, which can effect large changes in the region attended. On the basis of experience with the size and spacing of array elements, an observer scales the attentional focus to a gradient density that is optimal for the array characteristics. Subsequently or concurrently, this scaled attentional focus is directed to regions of the array at a rate of $50-80 \mathrm{msec}$ per element (Treisman \& Gelade, 1980). On some trials, the target can be detected by attention alone, and no saccades are made (Caggiano, Greenwood, \& Parasuraman, 2001; Zelinsky \& Sheinberg, 1997). Even when the target cannot be detected by attention alone, typically fewer than two saccades are made before the target is detected (Previc, 1995). Each saccade will land in the vicinity of the nearest neighbor distractor possessing a target propertyfor example, color (Motter \& Belky, 1998; Zelinsky, Rao, Hayhoe, \& Ballard, 1997) — but outside the region covered by the initial position of the attentional focus (Motter \& Belky, 1998). Work from our laboratory (Caggiano et al., 2001) and elsewhere (Previc, 1995; Zelinsky et al., 1997) has shown that search is faster when saccades are not made. Even when no saccades are made, search speed is subject to cue-induced modulation of attentional scale. Although little work has been done to investigate the ef- fects of attentional scaling on number and accuracy of saccades, our work has shown that, consistent with the reaction time (RT) data, numbers of saccades increase with cue size (Caggiano et al., 2001). This indicates that the processing component of attentional scaling plays an important role in the progress of search, although it has been little studied, as compared with the processing component of attentional shifting.

From this evidence, a picture begins to emerge of deployment of visuospatial attention as analogous to foveation of the eyes. Although visuospatial attention has a lower resolution than does the fovea (Intriligator \& Cavanagh, 2001), it can be brought to bear on a location more rapidly. Saccadic eye movements typically require $200 \mathrm{msec}$ to be initiated (Carter, Obler, Woodward, \& Albert, 1983), whereas shifts of attention can be executed in $50-80 \mathrm{msec}$ (Treisman \& Gelade, 1980). Moreover, vision is not suppressed during shifts of attention, as it is during saccades (Diamond, Ross, \& Morrone, 2000; Matin, 1974). Finally, visuospatial attention has the property of scaling, which, in contrast to the fovea, allows a broad or narrow region of space to be scanned. With these advantages over foveation, the focus of visuospatial attention can guide the oculomotor system by scouting for potential targets. This reiterated sequence of processes characterizes visual search.

In the present work, we sought confirmatory evidence for this model of visual search, which predicts that for a given set of processing demands, there exists an optimal scale of the attentional focus. Specifically, when precues are valid, the scale of the attentional focus will be finely modulated according to task demands. To date, the effects of cue size on visual search have been investigated over only a small range of two (Eriksen \& St. James, 1986) or three (Greenwood \& Parasuraman, 1999) cue sizes. The model also predicts that when precues are invalid, the optimal attentional scale will be large, but with resources distributed so as to facilitate detection of the target at a distance from the cue. Because invalid cues require attentional shifting as well as scaling, search RT will be slower following invalid, as compared with valid, cues. To test these predictions, in Experiment 1 we investigated the effect of target-distractor similarity, cue size, and cue validity on attentional scaling. This design extended our previous study of attentional scaling (Greenwood \& Parasuraman, 1999) by increasing the range of cue sizes and fully manipulating cue validity in both feature and conjunction searches. This allowed a test of the predictions of the model concerning the role of target-distractor similarity, cue validity, and cue size.

Implicit in our model is the notion that attentional scaling following an area cue is automatic and not strategic. This assumption bears on whether attentional scaling is a fundamental component process of visuospatial attention. Therefore, a possible role for strategy in attentional scaling must be considered. Several studies in which the LaBerge flanker task has been used to induce a narrow or broad attentional focus (LaBerge et al., 1991) have attributed age-related increases in attentional scaling to differ- 
ences in strategy (Gottlob \& Madden, 1999; Hartley, Kieley, $\&$ McKenzie, 1992). In size-cuing paradigms, a conscious strategy of searching inside the cue first would lead to faster search with smaller cues, because cues approaching element size contain fewer items and less space through which to search. Use of a strategy would be facilitated by the presence of the cue in the search array. However, assuming that the cue remains visible during array presentation, a strategy would not depend on the length of time the cue is visible before array onset. In contrast, a process of automatic scaling would be predicted to have a time course, much as the development of attentional shifting effects following a location cue has been shown to have a time course (Müller \& Rabbitt, 1989). The development of scaling effects over time from cue onset has not been fully investigated. Greenwood and Parasuraman (1999) observed that the effect of cue size was reduced when the interval between the cue and the array was brief. However, that observation was not made directly in a single experiment but, rather, in a comparison of effects across experiments with different participants. Nor was a range of cue-array intervals examined to allow detailed analysis of the temporal course of attentional scaling. These questions were addressed in Experiment 2.

The model also predicts that to the extent that attentional scaling is inherent in visual search processes, its role would be diminished in the absence of distractors. Single-unit studies have shown that attentional modulation of neuronal activity is stronger in the presence than in the absence of distractors (Motter, 1993; Noesselt et al., 2002). This effect is consistent with the interpretation that attention performs a filtering function (Luck \& Hillyard, 1994). Of the few existing studies of attentional scaling, most have used displays containing at least a few distractors (Eriksen \& St. James, 1986; Greenwood \& Parasuraman, 1999; LaBerge, Carlson, Williams, \& Bunney, 1997). However, one group of studies has shown scaling in the absence of distractors (Castiello \& Umiltà, 1990), although with effects that were relatively small. Whether attentional scaling occurs in the absence of distractors was investigated in Experiment 3.

The three-component model assumes that attentional scaling is driven by array characteristics of size and targetdistractor similarity. In virtually every previous study in which cues were used to induce attentional scaling, the cues indicated the size of a region of space, rather than the size of the target. Yet, as in the example at the beginning of this article, target size is an important feature of an object. Anyone who has missed seeing an approaching motorcycle while scanning for car traffic has experienced the effect of anticipated object size as a cue. Is attentional scaling carried out differently when the size of a target is cued? This is an important question, since it bears on whether scaling effects are relevant to the region to be searched or to the size of the target. It was examined in Experiment 3.

In summary, three experiments were conducted to test this model and, thereby, more fully investigate the role of attentional scaling in visual search. In the first experiment, we sought to confirm the previous finding of an effect of precue size on search speed and its alteration by aging, and to extend that finding to a large range of cue sizes, as well as invalidly located cues. In the second experiment, we assessed the role of strategy in cue size effects by manipulating cue duration and measuring the time course of cue size effects. In this experiment, we also investigated attentional scaling over the course of adult aging from young individuals to young-old individuals to old-old individuals. In the third experiment, we sought to investigate (1) the role of distractors in the deployment of attentional scaling in visual search and (2) the effect of the nature of cue informativeness on attentional scaling.

\section{EXPERIMENT 1}

This experiment was designed to investigate control of scaling of the attentional focus and the effects of age on that control. It was predicted that incremental increases in cue size would induce a corresponding expansion in the attentional focus, which would result in linear increases in RT. Previous studies had manipulated the cued region over two (Eriksen \& St. James, 1986) or three (Greenwood \& Parasuraman, 1999) sizes, with nonlinear cue size effects observed when three values were used. More than three data points would allow a better characterization of the function. In addition, effects of invalid cuing were assessed. On the basis of our three-component model, it was predicted that when cues were invalidly located, search would be fastest following a large cue (encompassing a number of array elements) than following a small cue (encompassing one element), consistent with the view that detection of a target at the periphery of the focus of attention would be facilitated by a larger attentional scale. Therefore and conversely, search following invalidly located precues would be slowest when the precues were small (element sized). Consistent with our previous finding, both feature and conjunction search speed would be modulated by cue size, but with stronger effects on conjunction search. The phenomenon of popout, which characterizes feature search, reduces the need for a constricted attentional focus. Finally, on the basis of previous work, it was predicted that age would increase cue-induced attentional scaling.

It should be noted that the descriptors small and large in referencing cue size are terms of convenience. More important than the actual size of a cue is its relation to the size of the target. In this work, a small cue is one large enough to encompass only one element. Larger cues are defined by the number of array elements they encompass.

\section{Method}

\section{Participants}

There were two groups of participants: 15 college student volunteers, 18-25 years of age, who were fulfilling course requirements by their participation, and 15 community-dwelling volunteers 65 years of age or older. The older volunteers were recruited by newspaper advertisements and were screened for health problems by in- 
Table 1

Demographics of Participant Groups (Means and Standard Deviations)

\begin{tabular}{|c|c|c|c|c|c|c|c|c|c|c|c|c|c|c|}
\hline \multirow[b]{3}{*}{ Group } & \multirow{2}{*}{\multicolumn{2}{|c|}{ Gender }} & \multirow{2}{*}{\multicolumn{2}{|c|}{ Age (Years) }} & \multirow{2}{*}{\multicolumn{2}{|c|}{ Education }} & & & \multicolumn{4}{|c|}{ Logical Memory (WMS) } & \multirow{2}{*}{\multicolumn{2}{|c|}{ Mini-Mental Stat }} \\
\hline & & & & & & & \multicolumn{2}{|c|}{ WAIS Vocabulary } & \multicolumn{2}{|c|}{ Immediate } & \multicolumn{2}{|c|}{$\overline{\text { Delayed }}$} & & \\
\hline & $\mathrm{M}$ & $\mathrm{F}$ & $M$ & $S D$ & $M$ & $S D$ & $M$ & $S D$ & $M$ & $S D$ & $M$ & $S D$ & $M$ & $S D$ \\
\hline \multicolumn{15}{|c|}{ Experiment 1} \\
\hline Young & 24 & 18 & 20.8 & 3.40 & 13.6 & 1.7 & 51.8 & 6.0 & 13.6 & 3.6 & 12.7 & 3.5 & - & - \\
\hline Young-old & 17 & 25 & 68.8 & $2.60 *$ & 14.6 & 2.4 & 62.3 & $4.3^{*}$ & 11.8 & 4.0 & 10.6 & 3.7 & 29.7 & 0.5 \\
\hline \multicolumn{15}{|c|}{ Experiment 2} \\
\hline Young & 5 & 11 & 18.2 & 0.67 & 12.5 & 0.9 & 54.9 & 7.5 & 16.9 & 3.2 & 15.9 & 3.4 & - & - \\
\hline Young-old & 5 & 11 & 68.2 & 2.60 & 14.9 & 3.2 & 60.6 & 5.0 & 14.4 & 2.9 & 11.9 & 4.0 & 29.4 & 0.7 \\
\hline Old-old & 4 & 12 & 78.1 & 2.50 & 14.2 & $2.5^{*}$ & 60.8 & $5.8^{*}$ & 12.3 & $3.5^{*}$ & 10.4 & $4.0^{*}$ & 29.1 & 1.1 \\
\hline \multicolumn{15}{|c|}{ Experiment 3} \\
\hline Young & 5 & 10 & 19.8 & 3.20 & 13.5 & 1.9 & 44.9 & 8.5 & 10.8 & 3.9 & 9.6 & 4.4 & & \\
\hline Young-old & 9 & 6 & 68.0 & 2.80 & 15.7 & 3.8 & 58.6 & 6.6 & 10.9 & 2.1 & 8.7 & 2.8 & & \\
\hline Old-old & 5 & 5 & 79.2 & 3.80 & 15.7 & $2.7^{*}$ & 62.1 & $3.9^{*}$ & 9.7 & 3.2 & 8.9 & 2.4 & & \\
\hline
\end{tabular}

Note-The Mini-Mental State Exam was not administered to young individuals. * Significant overall main effect of group $(p<.05)$, but no significant difference between young-old and old-old groups on a post hoc test.

terview and questionnaire. The demographic data appear in Table 1. All the participants had corrected visual acuity of at least 20/40 on a Rosenbaum Pocket Vision Screener.

\section{Stimuli}

Figure 1A illustrates the stimuli and procedures. Arrays were composed of 18 letters, arranged in three rows of 6 letters $\left(7.97^{\circ} \times\right.$ $\left.4.48^{\circ}\right)$. Rectangular precues enclosed 1 letter $\left(1.43^{\circ} \times 1.33^{\circ}\right)$, 4 letters $\left(3.05^{\circ}\right.$ on each side $), 6$ letters $\left(1.34^{\circ} \times 4.29^{\circ}\right), 9$ letters $\left(4.38^{\circ} \times\right.$ $\left.5.04^{\circ}\right)$, or 12 letters $\left(7.59^{\circ} \times 5.14^{\circ}\right)$. Viewing distance was $60 \mathrm{~cm}$. The letters were R, N, or G, drawn in one of three colors (green, blue, or pink), and were about $1.1^{\circ} \times 0.9^{\circ}$ of visual angle. The target letter was a pink R, present on $50 \%$ of the trials. The order and position of the letters in the arrays were chosen randomly, with the exception that on feature search trials only the target was pink, whereas on conjunction trials distractors were pink as well. Thus, on feature trials, the pink target appeared to pop out of the blue and green array of distractors. Popout was not experienced on conjunction trials. All the arrays were centered on the monitor. The arrays were constructed using a random order to select each letter and color, with the constraint that no more than one target could appear in each array. The cues were also placed randomly within the array.

It should be noted that the term conjunction search is employed in order to be consistent with current usage. It is not meant to imply that the requirement to search for a target defined by a conjunction of features is an important factor in search speed. Duncan and Humphreys (1989) have shown that target-distractor similarity is more important than the conjunction of features in inducing popout and producing slow or fast search.

Cue validity was defined according to the appearance of the target inside the cue. Therefore, on target-present trials, cues could be valid, with the target inside the cue (placed randomly), or invalid, with the target outside the cue (placed randomly). Within-subjects experimental conditions were target presence (present or absent), task type (feature or conjunction search), cue validity (valid, invalid, or neutral in predicting target location), and cue size (rectangular cues enclosing $1,4,6,9$, or 12 letters). Neutral cues $\left(7.88^{\circ} \times 4.67^{\circ}\right)$ enclosed the entire array. The size of the neutral cue was determined by pilot testing, using the size that produced an RT intermediate between those following valid and invalid cues.

The design could not be completely crossed because, when the target was absent, a cue could be neither valid nor invalid. Also, since neutral cues were defined by size, they could not vary in size, as did valid and invalid cues.

\section{Procedure}

The task required a speeded decision about the presence or absence of a feature or conjunction target (pink R) embedded in an 18letter array. Following a fixation point (1-sec duration), the screen was cleared, and a location precue appeared for the duration of the stimulus onset-asynchrony (SOA) and before the array of letters appeared for 2,000 msec. Future target location was indicated by a rectangular precue, which was valid (enclosed the target on $60 \%$ of the trials), invalid (enclosed letters other than the target on $20 \%$ of the trials), or neutral (enclosed the entire array on $20 \%$ of the trials). For each combination of SOA and precue size, there were 60 valid, 20 invalid, and 20 neutral trials. The precue appeared with a 500-msec cue-array SOA and remained visible until the end of the trial. The target was present on $50 \%$ of the trials. Therefore, depending on precue size, 1, 4, 6, 9, or 12 letters appeared inside the precue once the search array had appeared after the 500-msec SOA. On valid trials in which the cue was small (element sized), no shifting and no additional scaling was required once the target had appeared. On invalid trials in which the cue was small, only attentional shifting was required. The task was presented over six blocks, each of which lasted about 10 min and contained a randomly selected mixture of all the experimental conditions. All the conditions were presented in six mixed blocks. The participants were allowed to rest between each block and were required to rest for 10 min between Blocks 3 and 4 .

At the beginning and end of each testing session, a simple RT (SRT) task was administered. This consisted of 50 trials with a fixation point presented in the center of the screen for $500,1,000$, or $1,500 \mathrm{msec}$ (selected randomly on each trial) and followed by the letter $\mathrm{X}$ for $1,000 \mathrm{msec}$. The participant was required to make a speeded buttonpress response to the $\mathrm{X}$.

\section{Results}

In all repeated measures analyses carried out in this study, $F$ values were corrected for violations from sphericity by adjusting degrees of freedom, using the GreenhouseGeisser method.

\section{Accuracy}

Accuracy (see Table 2) ranged from $88.3 \%$ (in the young group on conjunction search with invalid cue size 1) to $98.7 \%$ (in the old group on feature search, invalid cue sizes 1, 4, and 12) but did not differ across age groups. 

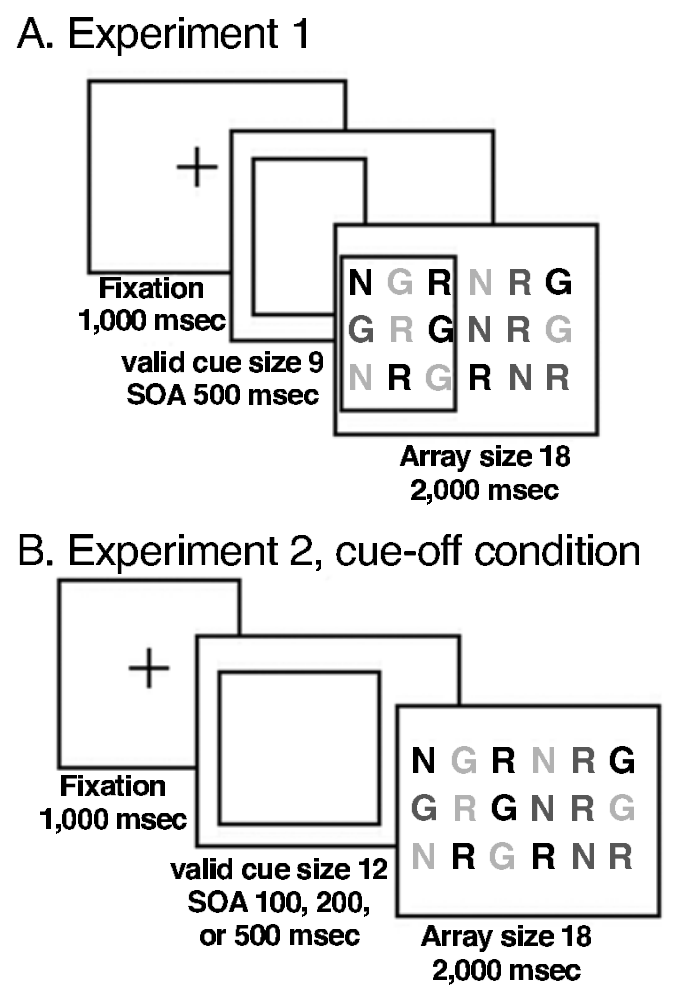

\section{Experiment 3, CUE AT CUE AT INVALID VALID LOCATION, INVALID SIZE LOCATION, INVALID SIZE}

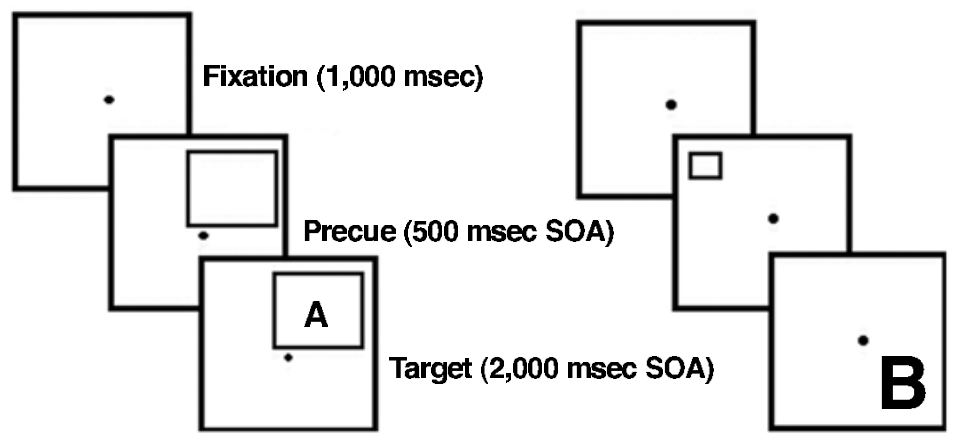

Figure 1. Illustration of the tasks used in each study. (A) Experiment 1. (B) Experiment 2; the target is represented by the light gray R. (C) Experiment 3.

Feature search was more accurate than conjunction search overall $[F(1,26)=10.20]$. However, feature search was more accurate when the cues were invalid, whereas conjunction search was more accurate when the cues were valid $[F(1,26)=4.40, p<.05]$. Accuracy increased with cue size on feature search but decreased with cue size on conjunction search $[F(4,104)=3.97, p<.01]$.

\section{Reaction Time}

To adjust for possible age-related slowing, each participant's RT was divided by the mean of the pre- and the postsession SRT medians. This method corrects for the portion of task-related RTs that arise from response speed
(Faust \& Balota, 1997). These RT ratios were used in all the following analyses, including the derived measures.

Omnibus analysis. When the target was present, there were main effects of age group $[F(1,28)=4.52, p<.05]$, $\operatorname{task}[F(1,28)=63.43, p<.0001]$, cue validity $[F(1,28)=$ $113.13, p<.0001]$, and cue size $[F(4,112)=9.37, p<$ $.0001]$ and an interaction of cue size and age group $[F(4,112)=4.80, p<.01]$. There were also interactions with task, including validity $\times$ task $\times$ age group $[F(1,28)=$ $4.60, p<.04]$ and validity $\times$ task $\times$ cue size $[F(4,112)=$ $10.99, p<.0001]$. Justified by the main effect of validity and the interactions of validity and cue size $[F(4,112)=$ $22.37, p<.0001]$, and by previous work showing that the 
Table 2

Experiment 1: Search Accuracy Mean Ratios

\begin{tabular}{lccccc}
\hline & \multicolumn{5}{c}{ Cue Size } \\
\cline { 2 - 6 } Task & 1 & 4 & 6 & 9 & 12 \\
\hline Conjunction & & & & & \\
$\quad$ Valid & .962 & .937 & .96 & .934 & .938 \\
$\quad$ Invalid & .914 & .923 & .93 & .933 & .949 \\
Feature & & & & & \\
$\quad$ Valid & .968 & .964 & .96 & .961 & .962 \\
$\quad$ Invalid & .967 & .966 & .96 & .973 & .967 \\
\hline
\end{tabular}

effects of cue size take a different form following valid, as compared with invalid, cues (Greenwood \& Parasuraman, 1999; Greenwood et al., 1997), valid and invalid data were subsequently analyzed separately. This allowed further examination of cue size $\times$ age group interactions.

Validly cued trials. When the cues were valid, search RT was faster (RT ratios smaller) on feature than on conjunction search trials (Figure 2A, Table 3). Consistent with our earlier work, both feature and conjunction search RTs were speeded by decreased cue size, but with a greater effect on conjunction search.

The benefit of valid cues, relative to neutral cues, can be calculated as the difference in RT ratios between valid and neutral cue conditions (neutral - valid RTs). In feature search, benefits decreased with cue size $[F(4,112)=14.35$, $p<.0001$ ], with the decrease being similar in young and old participants. In conjunction search (Figure 2B), benefits decreased with increasing cue size $[F(4,112)=34.26$, $p<.0001]$ but were greater in the old than in the young group $[F(1,28)=4.15, p<.05]$.

Since the effect of valid precue size on search speed was roughly linear, slopes of RT/cue-size functions were calculated by regressing RT from valid trials against cue size for each participant. Thus, the slope reflects the increase in RT with each increase in cue size. As can be seen in Figure 2A and Table 4, slopes were steeper in the old than in the young group $[F(1,28)=7.15, p<.01]$. Slopes were also steeper in conjunction than in feature search $[F(1,28)=$ $55.68, p<.0001]$, particularly in the older individuals [age group $\times$ task, $F(1,28)=7.01, p<.02$ ].

Invalidly cued trials. When cues were invalid - that is, when the target appeared outside the cue-RT was slowed. On invalidly cued trials (Figure 3A, Table 5), despite correction for age-related slowing, age group differences were not eliminated. RT ratios were significantly smaller in the young than in the old group, overall. Ratios were also significantly smaller on feature than on con-

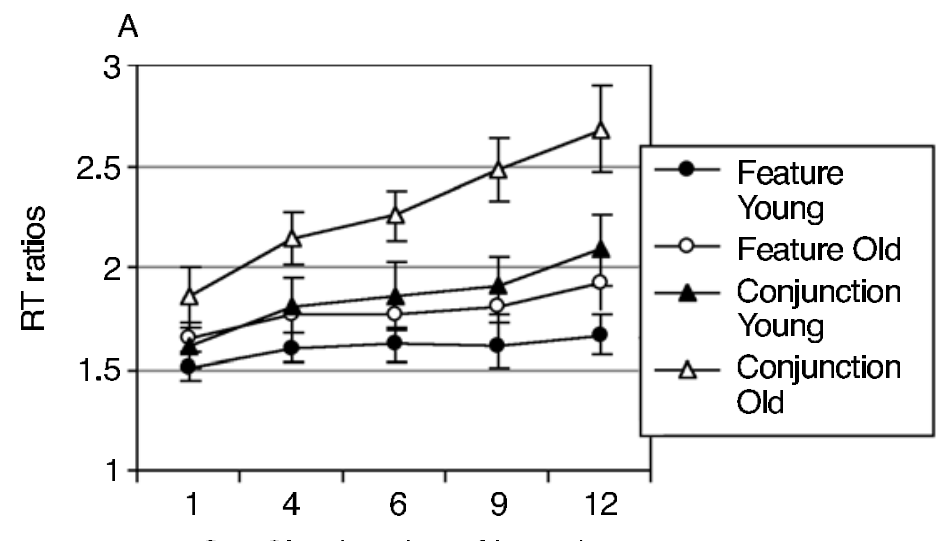

Cue Size (number of letters)

B

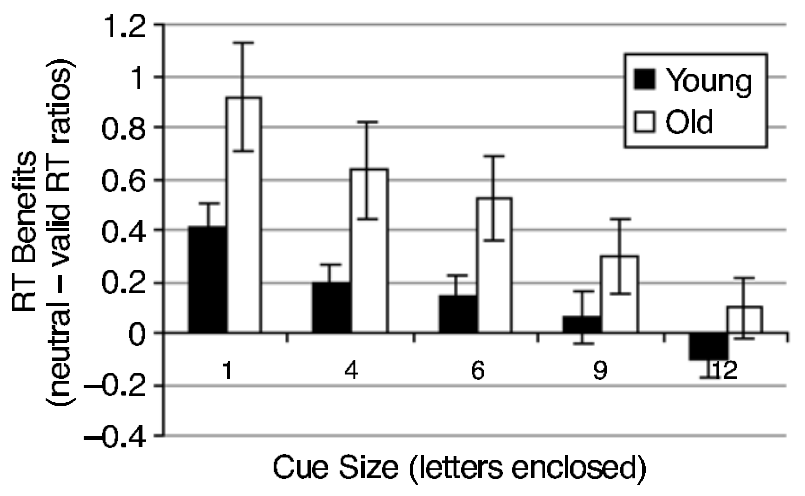

Figure 2. Experiment 1. (A) Search reaction time (RT) ratios plotted as a function of valid cue size for young and old groups under feature and conjunction search conditions. (B) Calculated benefits of valid cues (neutral valid RTs) on search RT plotted as a function of cue size for young and young-old groups. 
Table 3

Experiment 1: Analysis of Variance of Reaction Time Ratios From Validly Cued Feature and Conjunction Search Tasks

\begin{tabular}{llcccc}
\hline \multicolumn{1}{c}{ Source } & $d f$ & $F$ & $M S_{\mathrm{e}}$ & $\lambda$ & $p$ \\
\hline Between Subjects & & & & & \\
$\quad$ Age group (AG) & 1,28 & 3.84 & 1.83 & 3.838 & .0601 \\
Within Subjects & & & & & \\
Cue size (CS) & 4,112 & $50.91^{*}$ & 0.086 & 203.618 & .0001 \\
CS $\times$ AG & 4,112 & $5.57^{*}$ & 0.086 & 22.285 & .0004 \\
Task (T) & 1,28 & $15.35^{*}$ & 0.020 & 15.35 & .0005 \\
T $\times$ AG & 1,28 & 0.708 & 0.020 & 0.708 & .4072 \\
CS $\times$ T & 4,112 & $3.58^{*}$ & 0.036 & 14.311 & .0087 \\
CS $\times$ T $\times$ AG & 4,112 & 0.055 & 0.036 & 0.218 & .9944 \\
\hline$* p<.05$. & & & & &
\end{tabular}

junction search. Age and cue size both had a greater effect on conjunction search than on feature search. In Figure $3 \mathrm{~A}$, it can be seen that, on both feature and conjunction search trials, young, but not old, individuals were relatively slowed when the invalid cue was similar in size to the array elements. This produced a significant cue size $X$ age group interaction (Table 5).

In feature search, RT cost, the increase in RT when the cue was invalid relative to neutral (invalid - neutral RT ratios), was greater in the young group at the smallest cue but greater in the old group at all larger cue sizes [cue size $X$ age group, $F(4,112)=3.27, p<.03$ ], so that whereas costs decreased sharply with cue size in the young group, they increased with cue size in the old group.

In conjunction search, costs of invalid cues showed a more complex pattern (Figure 3B). The main effect of cue size $[F(4,112)=3.84, p<.01]$ and the interaction between age and cue size $[F(4,112)=2.80, p<.03]$ reflect the substantial cost arising from invalid element-sized cues experienced by the young group, but not by the old. Young and old participants differed little in the total effect of cue validity of the smallest cue (young $=1.244$; old $=$ 1.330 ), but the young group showed greater costs of invalid cues (young $=0.831$; old $=0.413$ ), whereas the old group showed greater benefits (young $=0.413$; old $=$ $0.917)$.

\section{Discussion}

In concert with predictions of the model and consistent with previous findings (Greenwood \& Parasuraman, 1999), decreasing the size of valid location precues toward the size of array elements speeded search, whether the search was for single features under popout conditions or for conjunctions of features. Effects of cue precision were stronger in conjunction than in feature search, with older individuals obtaining greater benefits than young from valid precise cues to target location. As cue size increased-and the precision of the information about target position accordingly decreased - this benefit decreased as well, more precipitously in the old participants than in the young (Figure 2B). Therefore, not only was the ability to benefit from the precision of prior information preserved in aging, but also the benefit was greater in the older individuals.

The finding of cue size effects on popout is consistent with some previous studies of cue location effects on popout (Prinzmetal, Presti, \& Posner, 1986; Theeuwes, Kramer, \& Atchley, 1999). However, others have shown either little (Nakayama \& Makeben, 1989) or no (Braun \& Sagi, 1991) effect of cue location on popout.

When cues were invalid for location, the optimal scale of attention changed. The level of costs of invalid cues approached the level of benefits of valid cues, but only in the young individuals at the smallest cue. Overall, the young participants experienced greater costs of invalid cues, whereas the old participants experienced greater benefits of valid cues. On invalid trials, when the cue was small,

Table 4

Slope Values of Cue-Size/RT Functions

(Means and Standard Errors)

\begin{tabular}{|c|c|c|c|c|c|c|}
\hline \multirow[b]{3}{*}{ Condition } & \multicolumn{4}{|c|}{ Search Task } & & \\
\hline & \multicolumn{2}{|c|}{ Feature } & \multicolumn{2}{|c|}{ Conjunction } & & \\
\hline & $M$ & $\overline{S E}$ & $M$ & $\overline{S E}$ & & \\
\hline \multicolumn{7}{|c|}{ Experiment 1} \\
\hline Young & 2.62 & 1.01 & 9.86 & 2.14 & & \\
\hline Old & 5.55 & 1.51 & 20.74 & 3.16 & & \\
\hline \multicolumn{7}{|c|}{ Experiment 2} \\
\hline & \multicolumn{6}{|c|}{ Group } \\
\hline & \multicolumn{2}{|c|}{ Young } & \multicolumn{2}{|c|}{ Young-Old } & \multicolumn{2}{|c|}{ Old-Old } \\
\hline & $M$ & $S E$ & $M$ & $S E$ & $M$ & $S E$ \\
\hline \multicolumn{7}{|c|}{ Feature Search } \\
\hline SOA 100 & 1.60 & .60 & 1.06 & .64 & -0.45 & .47 \\
\hline SOA 200 & 4.05 & .54 & 2.43 & .63 & 2.04 & .68 \\
\hline SOA 500 & 5.42 & .80 & 3.54 & .78 & 0.22 & .65 \\
\hline \multicolumn{7}{|c|}{ Conjunction Search } \\
\hline SOA 100 & 6.80 & 1.05 & 6.49 & 2.07 & 2.18 & 1.45 \\
\hline SOA 200 & 10.06 & 1.32 & 20.90 & 2.14 & 13.52 & 2.43 \\
\hline SOA 500 & 10.23 & 1.32 & 19.25 & 2.14 & 8.87 & 1.78 \\
\hline
\end{tabular}



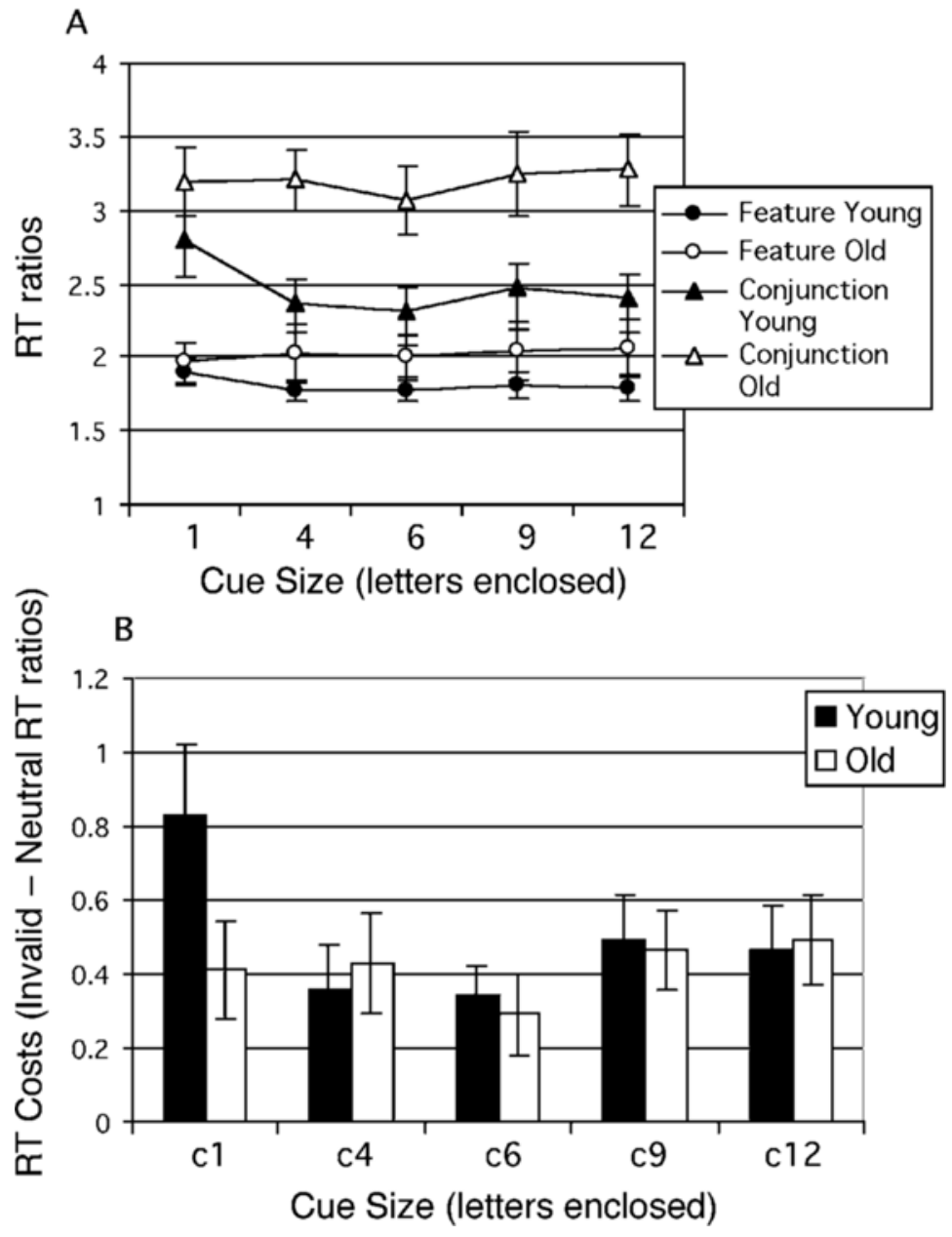

Figure 3. Experiment 1. (A) Search reaction time (RT) ratios plotted as a function of invalid cue size for young and old groups under feature and conjunction search conditions. (B) Calculated benefits of valid cues (invalid - neutral RTs) on search RT plotted as a function of cue size for young and young-old groups.

the location of the target outside the cue slowed search, particularly in young participants. This marked increase in costs in the young participants, associated with elementsized cues, may be comparable to the disengagement seen in cued discrimination tasks on invalid trials. In both cases, attention is focused on a discrete region of space, and the target appears elsewhere. This raises the question (posed by a reviewer) of why the older group showed costs of invalid cues that were lower overall than those seen in the young group. This result can be compared with the previously reported disengagement effect (slowed RT following invalid cues) in old-old individuals in a nonsearch discrimination task (Greenwood \& Parasuraman, 1994). In light of this, why did the older group in this task not show slowed disengagement following invalid cues? The previous finding of slowed disengagement was seen in old-old, but not in young-old, individuals, and Experiment 1 tested only young-old individuals. It can also be speculated that the more diffuse attentional focus in the older group may have actually reduced the costs associated with invalidly located cues. As evidence for this, note the similar level of costs for young and old individuals when cues encompassed more than one array element (Figure 3B).

Table 5

Experiment 1: Analysis of Variance of Reaction Time Ratios From Invalidly Cued Feature and Conjunction Search Tasks

\begin{tabular}{lccccc}
\hline \multicolumn{1}{c}{ Source } & $d f$ & $F$ & $M S_{\mathrm{e}}$ & $\lambda$ & $p$ \\
\hline $\begin{array}{l}\text { Between Subjects } \\
\text { Age group (AG) }\end{array}$ & 1,28 & 4.75 & 3.50 & 4.75 & .0400 \\
Within Subjects & & & & & \\
$\quad$ Task (T) & 1,28 & $79.02^{*}$ & 0.801 & 79.019 & .0001 \\
Task $\times$ AG & 1,28 & $6.209^{*}$ & 0.801 & 6.209 & .0189 \\
Cue size (CS) & 4,112 & $3.280^{*}$ & 0.073 & 13.12 & .0139 \\
CS $\times$ AG & 4,112 & $3.798^{*}$ & 0.073 & 15.19 & .0062 \\
T $\times$ CS & 4,112 & $2.637^{*}$ & 0.051 & 10.55 & .0376 \\
T $\times$ CS $\times$ AG & 4,112 & 0.786 & 0.051 & 3.143 & .5367 \\
\hline
\end{tabular}

$* p<.05$. 
These results are consistent with our 1999 model in showing that the focus of visuospatial attention is flexibly and dynamically adjusted in response to prior information about a region in space where a target might appear. That cue size effects were greater on conjunction search than on feature search is also consistent with the GS model of Cave and Wolfe (1990). Although neither FIT nor GS predicts attentional scaling, GS states that top-down influences should be greater for conjunction search.

These results are also consistent with our model in showing that the optimal adjustment changes with task demands. When the target appears at the cued location (valid cues) and, thus, within the attentional focus, the optimal scale for searching is constricted around element size. When the target appears outside the cue (invalid cues) and, presumably, at the periphery of the attentional focus, the optimal scale appears to be expanded. In the young participants this was seen in facilitated search when the cue was large and invalid. These results suggest that young and old individuals may employ the information provided by precues differently. At cue onset, it cannot be known whether the cue will be valid. The young participants scaled their attention to the element-sized small cue but were slowed when the cue turned out to be invalid. In contrast, although the old individuals benefited from the small valid cues, they were not penalized as much as the young ones by small invalid cues. This suggests that although both young and old individuals may scale the attentional focus to cue size, older individuals may be less able than young ones to maintain such a constricted focus and may rapidly revert to a broader attentional focus.

Consistent with the model, this experiment (1) confirmed attentional scaling as a component of visuospatial attention in visual search, (2) revealed a fairly close relationship between precue size and search speed, (3) showed that whereas a constricted scale is optimal when cues are validly location, an expanded scale is optimal when cues are invalidly located, and (4) confirmed a heightened effect of attentional scaling with aging and extended that finding to a large range of precue sizes.

However, in both our model and Experiment 1, it is assumed that attentional scaling is not strategic. To examine this assumption, it is important to determine the extent to which strategy plays a role in attentional scaling effects.

\section{EXPERIMENT 2}

Having examined some of the predictions of the threecomponent theory, we next turned our attention to a key assumption of that theory-namely, that scaling of the attentional focus is automatic, rather than conscious and strategic. A strategy-based explanation could posit that precue size effects would not arise from attentional scaling to the appearance of the cue but, rather, to the order in which search was conducted once the array had appeared. According to this view, a conscious plan to initially search inside the cue would result in faster search with smaller cues. This was investigated in two ways: by manipulating cue duration and by measuring the time course of attentional scaling.

The rationale for manipulating cue duration is as follows. If cue size effects are strategic, the cue would be used as a guide during the process of search. Therefore, turning the cue off before the array appeared would likely weaken cue size effects on both feature and conjunction searches, since participants would need to consciously remember both the location and the size of the cue in order to use them to guide search once the array had appeared.

The rationale for measuring the time course of scaling is as follows. If cue size effects are strategic, carrying out the strategy could not begin before the appearance of the array. Therefore, the length of time the cue is visible prior to array onset (cue-array SOA) should have little effect, particularly if the cue remains visible during the presentation of the search array. Alternatively, if cue size effects are attentional and not strategic, they likely have a time course, as has been shown for cue location effects on detection and discrimination in nonsearch tasks (Greenwood, Parasuraman, \& Haxby, 1993; Müller \& Rabbitt, 1989). Therefore, in this experiment cue-target SOA was manipulated within subjects. In light of the apparently fully developed cuing effects observed at the 500-msec SOA in Experiment 1, two shorter SOAs were added in Experiment 2.

Finally, to examine in greater detail the impact of aging on these effects, both young-old and old-old groups of individuals were tested. In previous work, we have found that age exerts nonlineareffects on cuing dynamics, with effects of cue size on search speed increased in the young-old (65-74 years of age) but decreased in the old-old individuals (75-84 years of age; Greenwood \& Parasuraman, 1999). Adding an old-old group allowed the time course of development of cue effects to be measured over the course of adult aging.

Effects of attention are predicted to apply to both feature and conjunction searches, whereas effects of strategy are predicted to have no effect on feature search.

\section{Method}

\section{Participants}

There were 16 young college student volunteers and 32 older community-dwelling volunteers ranging in age from 65 to 84 years. The older individuals were divided by age into two groups of 16 young-old (65-74 years of age) and 16 old-old (75-84 years of age). Demographic characteristics of these groups are presented in Table 1.

\section{Stimuli}

The stimuli were identical to those in Experiment 1.

\section{Procedure}

The task required a speeded decision about the presence or absence of a feature or conjunction target (pink R) embedded in an 18letter array (Figure 1A). Target location was precued by a rectangle that varied in size, enclosing $1,4,6,9$, or 12 letters on a given trial. The precue was valid (enclosed the target) on $77 \%$ of the trials and invalid, with the target absent, on $23 \%$ of the trials. Cue validity was not a factor, in order to allow manipulation of SOA, cue duration, and cue size within subjects. The location precue preceded the array 
onset by an SOA of 100, 200, or $500 \mathrm{msec}$. On half the trials, the cue remained superimposed over the array until the end of the trial (cueon condition). On the other half, the cue was turned off when the array appeared (cue-off condition). Task conditions were presented in mixed blocks. In order to conduct this experiment within one testing session, the number of invalid trials had to be minimized, and therefore, cue validity was not included as a factor. For each combination of task type, cue duration, cue size, and SOA, there were 20 valid trials and 6 invalid trials. The between-subjects factor was age group. Within-subjec ts factors were task type (feature or conjunction), cue duration (cue on or cue off), cue size $(1,4,6,9$, or 12), and cuearray $\operatorname{SOA}(100,200$, or $500 \mathrm{msec})$. Repeated measures $F$ values were corrected for violations from sphericity by adjusting degrees of freedom, using the Greenhouse-Geisser method.

\section{Results}

\section{Accuracy}

Accuracy ranged from $88.4 \%$ to $100 \%$, being highest in the young-old group and lowest in the young group, but with no significant interactions with age group (Table 6). Feature search was more accurate than conjunction search $[F(1,45)=79.52, p<.0001]$, and accuracy declined with longer SOAs $[F(2,90)=10.13, p<.0001]$ and larger cue size $[F(2,90)=10.12, p<.001]$. There was no main effect of cue duration.

\section{Reaction Time}

Each participant's RT was divided by the mean of the pre- and postsession SRT medians to adjust for age-related slowing. An omnibus ANOVA was performed on these RT ratios from target-present valid cue RT conditions (the small number of invalid trials were not analyzed). This analysis revealed significance for all main effects [age group, $F(2,45)=8.19, p<.001 ;$ task $F(1,45)=263.87$, $p<.0001$; cue size, $F(4,180)=76.52, p<.0001$; cue duration, $F(1,45)=17.46, p<.0001 ;$ and SOA, $F(2,90)=$ $25.87, p<.0001]$. Since additional analyses are reported below, only the significant three-way interactions involving age group are reported here [task $\times$ cue size $\times$ age group, $F(8,180)=2.76, p<.02$; cue size $\times \mathrm{SOA} \times$ age group, $F(16,360)=1.9, p<.03]$. On the basis of the sig-

Table 6

Experiment 2: Accuracy Data Ratios

\begin{tabular}{|c|c|c|c|c|c|c|}
\hline \multirow[b]{2}{*}{ Task } & \multirow[b]{2}{*}{ SOA } & \multicolumn{4}{|c|}{ Cue Size } & \multirow[b]{2}{*}{12} \\
\hline & & 1 & 4 & 6 & 9 & \\
\hline \multicolumn{7}{|c|}{ Cue On } \\
\hline \multirow[t]{3}{*}{ Conjunction } & 100 & .958 & .960 & .949 & .940 & .938 \\
\hline & 200 & .951 & .948 & .931 & .951 & .939 \\
\hline & 500 & .923 & .950 & .933 & .923 & .927 \\
\hline \multirow[t]{3}{*}{ Feature } & 100 & .965 & .964 & .965 & .970 & .958 \\
\hline & 200 & .960 & .960 & .964 & .959 & .963 \\
\hline & 500 & .969 & .967 & .960 & .955 & .951 \\
\hline \multicolumn{7}{|c|}{ Cue Off } \\
\hline \multirow[t]{3}{*}{ Conjunction } & 100 & .963 & .971 & .969 & .949 & .971 \\
\hline & 200 & .949 & .957 & .953 & .952 & .959 \\
\hline & 500 & .960 & .956 & .945 & .947 & .952 \\
\hline \multirow[t]{3}{*}{ Feature } & 100 & .988 & .983 & .989 & .981 & .981 \\
\hline & 200 & .983 & .989 & .979 & .980 & .982 \\
\hline & 500 & .979 & .990 & .985 & .975 & .986 \\
\hline
\end{tabular}

nificant main effect of task and of previous work showing smaller effects of cue size on feature than on conjunction search (Greenwood et al., 1997), separate analyses were performed on each task type. This allowed separate examination of cue size $\times$ age group interactions for feature and conjunction search.

Feature search. Feature search RT ratios (Figure 4, Table 7) increased significantly with age and cue size but decreased with SOA. Effects of cue size were greater when the cue remained on (cue-on condition), with the two older groups more affected than the young group by manipulations of cue size and cue duration (cue size $X$ age group, cue duration $\times$ age group, and cue duration $\times$ cue size $X$ age group).

Effects of cue size developed with time from cue onset to array onset (SOA), whether or not cues remained on during array presentation (cue size $\times$ SOA). When only $100 \mathrm{msec}$ intervened between precue and array onsets, there was very little systematic effect of precue size on feature search speed, except in the young group when the cue remained on (cue-on condition in Figure 4D). Regardless of age, RT ratios tended both to decrease following the smallest cue and to increase following the largest cue, but with earlier development of cuing effects in the young group (Figures 4B, 4C, 4E, and 4F). The young and the young-old groups differed mainly in the amount of facilitation obtained from the smaller precues. In contrast, in the old-old group, RT was slowed by small and large cues but was facilitated by middle-sized cues. These effects were particularly pronounced when the cue remained on (cueon condition). As compared with the changes in the effect of the small and middle-sized cues, the effect of the largest cue changed relatively little with SOA in the older groups (cue duration $\times$ cue size $\times$ SOA $\times$ age group).

Because the effect of valid precue size on feature search speed was roughly linear, slopes of the RT/cue-size function were calculated for all the participants, using data from valid trials when the target was present (slopes in Table 4). Slope tended to increase with cue duration, but only at a marginal level of significance $[F(1,45)=3.64, p<.06]$. The three groups differed in slope [age group, $F(2,45)=$ $8.56, p<.001]$. A post hoc test (Tukey HSD) indicated that slopes of both the young and the young-old groups differed from those of the old-old group but did not differ significantly from each other $(p<.05)$. This age group effect was modified by SOA [SOA, $F(2,90)=14.06, p<$ .03 ; SOA $\times$ age group, $F(4,90)=2.88, p<.03$ ] . In both the young and the young-old groups, the RT/cue-size slopes increased with SOA, but in the old-old group, slopes first increased then decreased as SOA lengthened.

Conjunction search. On conjunction search trials (Table 8), RT increased with age and cue size. A Tukey HSD post hoc test revealed that RT ratios of the young group differed from those of both older groups $(p<.05)$, who did not differ significantly from each other. RT ratios were larger overall under the cue-off than under the cueon condition, and this effect of cue duration interacted with cue size, but not with SOA or group. RT was also fa- 

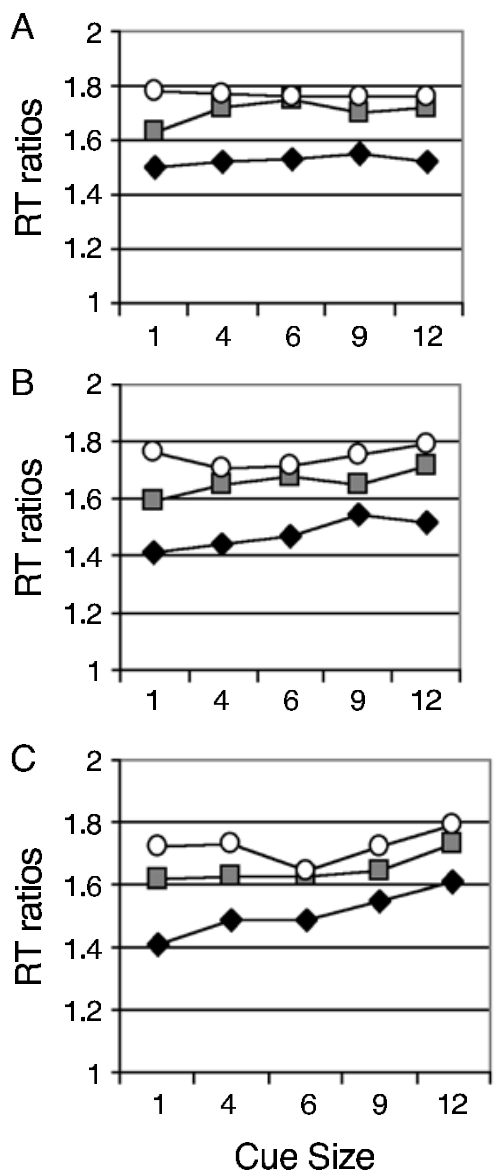
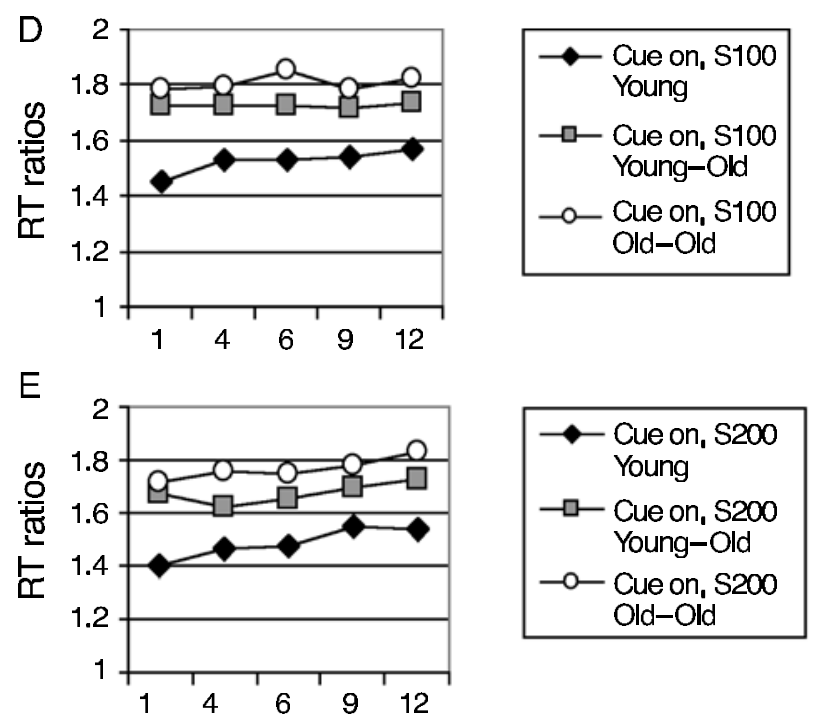

$\mathrm{F}$
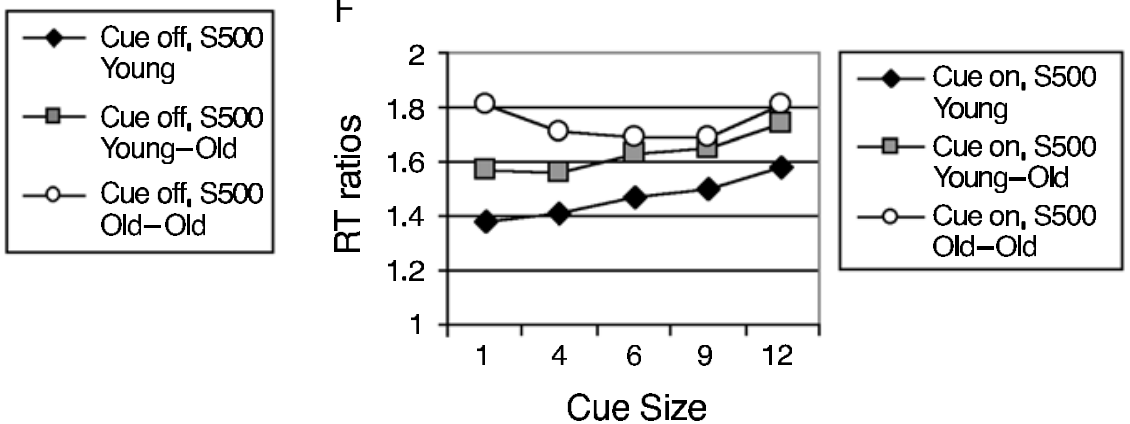

Figure 4. Experiment 2: feature search reaction time (RT) ratios plotted as a function of valid cue size and cue duration in young, young-old, and old-old groups. In panels A-C, the cue was turned off before the search array appeared: (A) cue-array stimulus onset asynchrony (SOA), $100 \mathrm{msec}$; (B) cue-array SOA, $200 \mathrm{msec}$; (C) cue-array SOA, $500 \mathrm{msec}$. In panels D-F, the cue remained on after the search array appeared: (D) cue-array SOA, $100 \mathrm{msec}$; (E) cue-array SOA, $200 \mathrm{msec}$; (F) cue-array SOA, $500 \mathrm{msec}$.

cilitated as SOA lengthened. As was seen with feature search, the effects of cue size developed with increased SOA, whether or not cues remained on during array presentation. There were no significant interactions involving age group and cue duration. In contrast to feature search, in conjunction search increases in cue size appeared to slow RT following the larger cues, rather than speeding RT following smaller cues (Figure 5). When only $100 \mathrm{msec}$ had elapsed between cue onset and target onset (100-msec SOA), RT was facilitated by smaller cue sizes but was little modulated by the larger cues. In contrast, when 200 or $500 \mathrm{msec}$ elapsed between cue onset and target onset, RT slowed with increasing cue size. This indicates that in the young group, only $100 \mathrm{msec}$ was needed to adjust the attentional focus to the smallest cue, whereas adjustment to the scale of the larger cues required more time. These factors interacted, so that the effects of cue size increased from the young to the young-old participants but then decreased in the old-old participants (Figure 5). The main age difference between the groups can be seen in the benefit obtained from the smaller cues. Both young and young- old groups showed pronounced facilitation of search speed at the smallest, relative to the middle-sized, cues, in contrast to a weaker effect of smaller cues in the old-old group.

The age groups also differed in the effect of SOA, with a smaller effect on the young than on the two older groups. Whereas the young-old group showed a fairly linear increase in RT with cue size at the longer SOAs, in the oldold group there was a linear increase only at the 200-msec SOA. By $500 \mathrm{msec}$ after cue onset, RT was slowed by smaller cues, as compared with the six-letter cue. This result is similar to that seen in Experiment 1 in the older group. Thus, in contrast to the scaling effect in the young and young-old groups, which increased linearly with time from cue onset, scaling in the old-old group followed a linear pattern by $200 \mathrm{msec}$, which had weakened by $500 \mathrm{msec}$. As compared with the $200-\mathrm{msec}$ SOA, by $500 \mathrm{msec}$ after cue onset the smaller cues appeared to slow search more, whereas the larger cues appeared to slow search less. This suggests that with increased time after the cue, search in old-old individuals is facilitated only when precues encompass several search items. 
Table 7

Experiment 2: Analysis of Variance of Reaction Time Ratios From Feature Search Task

\begin{tabular}{lrclrr}
\hline \multicolumn{1}{c}{ Source } & $d f$ & $F$ & $M S_{\mathrm{e}}$ & \multicolumn{1}{c}{$\lambda$} & \multicolumn{1}{c}{$p$} \\
\hline Between subjects & & & & & \\
$\quad$ Age group (AG) & 2,45 & 4.750 & 1.796 & 9.344 & .0143 \\
Within Subjects & & & & & \\
$\quad$ Cue duration (CD) & 1,45 & $3.014^{*}$ & 0.009 & 3.014 & .0894 \\
CD $\times$ new group (NG) & 2,45 & $4.664^{*}$ & 0.009 & 9.328 & .0144 \\
Cue size (CS) & 4,180 & $30.885^{*}$ & 0.01 & 123.418 & $<.0001$ \\
CS $\times$ AG & 8,180 & $5.887^{*}$ & 0.01 & 47.093 & $<.0001$ \\
SOA & 2,90 & $26.362^{*}$ & 0.015 & 52.723 & $<.0001$ \\
SOA $\times$ NG & 4,90 & 1.477 & 0.015 & 5.907 & .2159 \\
CD $\times$ CS & 4,180 & 1.289 & 0.007 & 5.157 & .2760 \\
CD $\times$ CS $\times$ AG & 8,180 & $2.047^{*}$ & 0.007 & 16.372 & .0434 \\
CD $\times$ SOA & 2,90 & $6.447 *$ & 0.006 & 12.895 & .0024 \\
CD $\times$ SOA $\times$ NG & 4,90 & 1.425 & 0.006 & 5.702 & .2320 \\
CS $\times$ SOA & 8,360 & $7.505^{*}$ & 0.008 & 60.037 & $<.0001$ \\
CS $\times$ SOA $\times$ NG & 16,360 & 1.216 & 0.008 & 19.457 & .2527 \\
CD $\times$ CS $\times$ SOA & 8,360 & 1.391 & 0.006 & 11.130 & .1988 \\
CD $\times$ CS $\times$ SOA $\times$ NG & 16,360 & $1.969^{*}$ & 0.006 & 31.512 & .0143 \\
\hline
\end{tabular}

Note-Values enclosed in parentheses represent mean square errors. $* p<.05$.

Slopes of the conjunction RT/cue-size function were calculated from valid trials when the target was present. As is evident in Figure 5 and Table 4, slopes increased with SOA overall $[F(2,90)=27.10, p<.0001]$. Slopes differed between groups $[F(2,45)=6.36, p<.004]$, being steepest in the young-old group and shallowest in the young group. The slopes of the young-old group differed significantly from those of both the young and the old-old groups, but those of the young and the old-old groups did not differ from each other (Tukey, $p<.05$ ). The effect of age interacted with SOA [age group $\times$ SOA, $F(4,90)=3.57, p<.01]$. Slopes generally increased with SOA in the young and young-old groups but showed a more biphasic pattern in the old-old group, in whom slope first increased, then decreased as SOA lengthened. Slopes were steeper overall when the cue remained on $[F(1,45)=$ $9.51, p<.004]$, but the age groups did not differ on this effect. Conjunction search slope was found to be negatively correlated with age for all older individuals at SOAs of $100(-.271), 200(-.209)$, and $500(-.431, p<$ $.01)$ msec. Figure 6 shows a scatterplot of age $\times$ slope for conjunction search at the 500-msec SOA.

\section{Discussion}

The effect of precue precision on search speed emerged only gradually with time from cue onset. This is interpreted as evidence against a role for strategy in cue size effects. A strategy would most reasonably be applied to the task as a whole. For a strategy to explain these results, it would be necessary that the participant (1) decide anew on every trial to employ a strategy, (2) having done so, prepare anew on every trial to employ the strategy, and (3) carry out those preparations during the SOA (as a reviewer suggested). Under the cue-on condition, the array and the cue were visible together for $2 \mathrm{sec}$, allowing ample time for a strategy to be used once the array had appeared. Never- theless, cue-target SOA clearly modulated cue size effects. Moreover, when the cue remained on during array presentation, there was close modulation of search RT by cue size with a $500-\mathrm{msec}$ SOA, but not with a 100 -msec SOA. It is hard to explain how a strategy could produce that result. Nor is it easy to explain why a strategy of searching first inside the cue would actually lead to slower search by older individuals when the cue was element sized (Figure 5F).

A strategy-based explanation could also posit that participants would rely on memory for the cued region and then search preferentially within that region. If cue size effects were due to iconic memory for the cue (as was suggested by a reviewer), they would be maximal at $200 \mathrm{msec}$ or so and then decline. Instead, cue size effects increased with time, particularly for the largest cue. It is not obvious why memory for the location and size of large cues would improve more than memory for small cues with time from cue onset. We conclude that although these results cannot conclusively rule out an effect of strategy, they are inconsistent with such an interpretation.

Feature and conjunction search were affected similarly by cue size manipulations, albeit with greater effects on conjunction search. This is also consistent with an attentionbased explanation, since a strategy would be unnecessary in the presence of popout. Effects of cue size on feature and conjunction search are discussed more fully in the General Discussion section.

Some benefit of the smaller cues was seen as early as $100 \mathrm{msec}$ after cue onset, but the cost of the larger cues continued to increase with SOA. In both feature and conjunction search, cue size effects were not fully developed until $500 \mathrm{msec}$ had elapsed after cue onset. This finding can be contrasted with the faster development $(<50 \mathrm{msec})$ of the effect of peripheral (exogenous) location cues in nonsearch paradigms (Greenwood \& Parasuraman, 1994; Müller \& Rabbitt, 1989).

Table 8

Experiment 2: Analysis of Variance of Conjunction Search Reaction Time

\begin{tabular}{lrccrr}
\hline \multicolumn{1}{c}{ Source } & $d f$ & $F$ & $M S_{\mathrm{e}}$ & \multicolumn{1}{c}{$\lambda$} & \multicolumn{1}{c}{$p$} \\
\hline Between subjects & & & & & \\
$\quad$ Age group (AG) & 2,45 & $10.059^{*}$ & 5.718 & 20.118 & .0002 \\
Within Subjects & & & & & \\
$\quad$ Cue duration (CD) & 1,45 & $25.934^{*}$ & 0.082 & 25.934 & $<.0001$ \\
CD $\times$ new group (NG) & 2,45 & 2.275 & 0.082 & 4.55 & .1145 \\
Cue size (CS) & 4,180 & $70.310^{*}$ & 0.100 & 281.24 & $<.0001$ \\
CS $\times$ NG & 8,180 & 2.595 & 0.100 & 20.759 & .0105 \\
SOA & 2,90 & $5.119 *$ & 0.061 & 30.238 & $<.0001$ \\
SOA $\times$ NG & 4,90 & $3.768^{*}$ & 0.061 & 15.072 & .007 \\
CD $\times$ CS & 4,180 & $4.695^{*}$ & 0.061 & 18.778 & .0013 \\
CD $\times$ CS $\times$ NG & 8,180 & 0.955 & 0.061 & 7.643 & .4725 \\
CD $\times$ SOA & 2,90 & 0.279 & 0.057 & 0.558 & .7573 \\
CD $\times$ SOA $\times$ NG & 4,90 & 1.463 & 0.057 & 5.852 & .2201 \\
CS $\times$ SOA & 8,360 & $15.617 *$ & 0.065 & 124.936 & $<.0001$ \\
CS $\times$ SOA $\times$ NG & 16,360 & $1.678^{*}$ & 0.065 & 26.846 & .0488 \\
CD $\times$ CS $\times$ SOA & 8,360 & 1.299 & 0.047 & 10.391 & .2427 \\
CD $\times$ CS $\times$ SOA $\times$ NG & 16,360 & 0.809 & 0.047 & 12.946 & .6754 \\
\hline
\end{tabular}

Note-Values enclosed in parentheses represent mean square errors. ${ }^{*} p<.05$. 

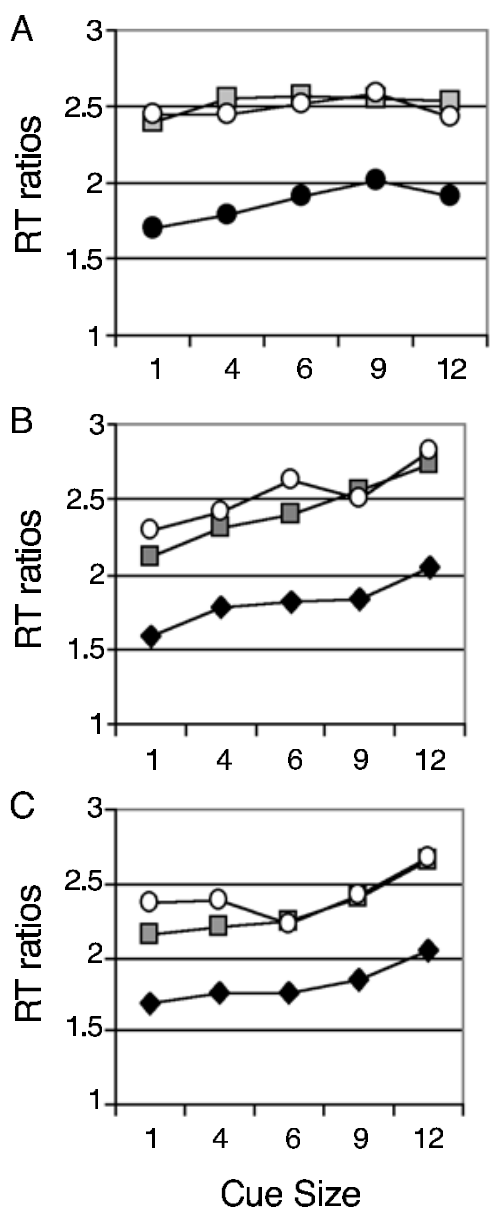
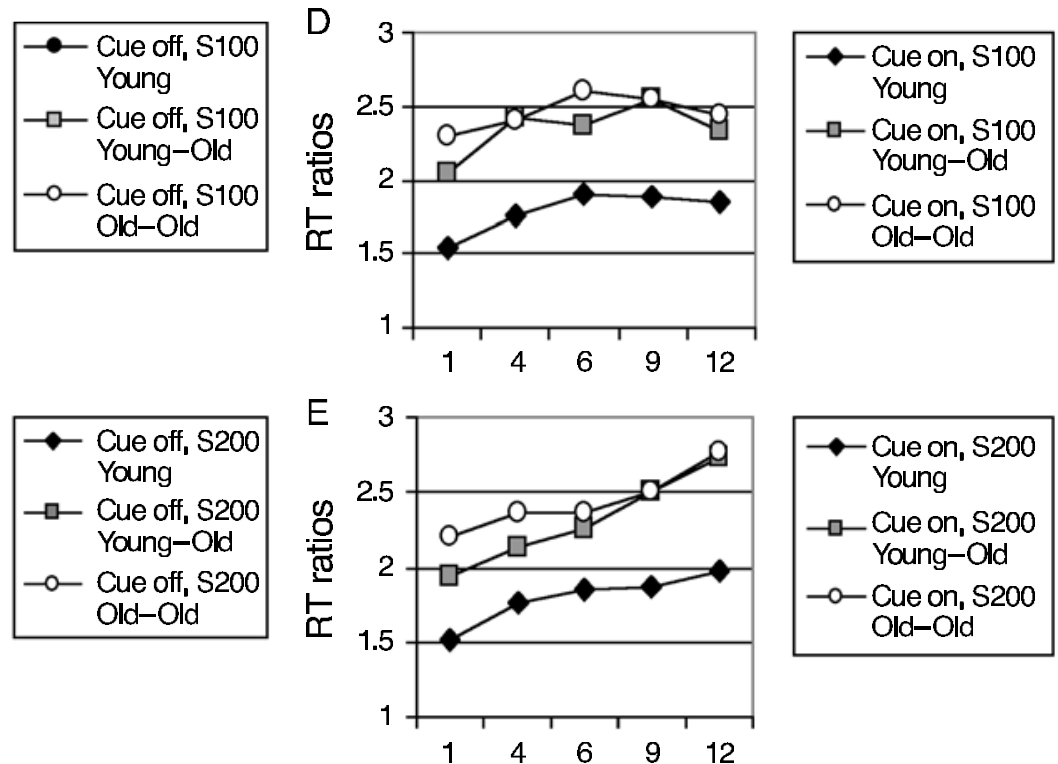

F
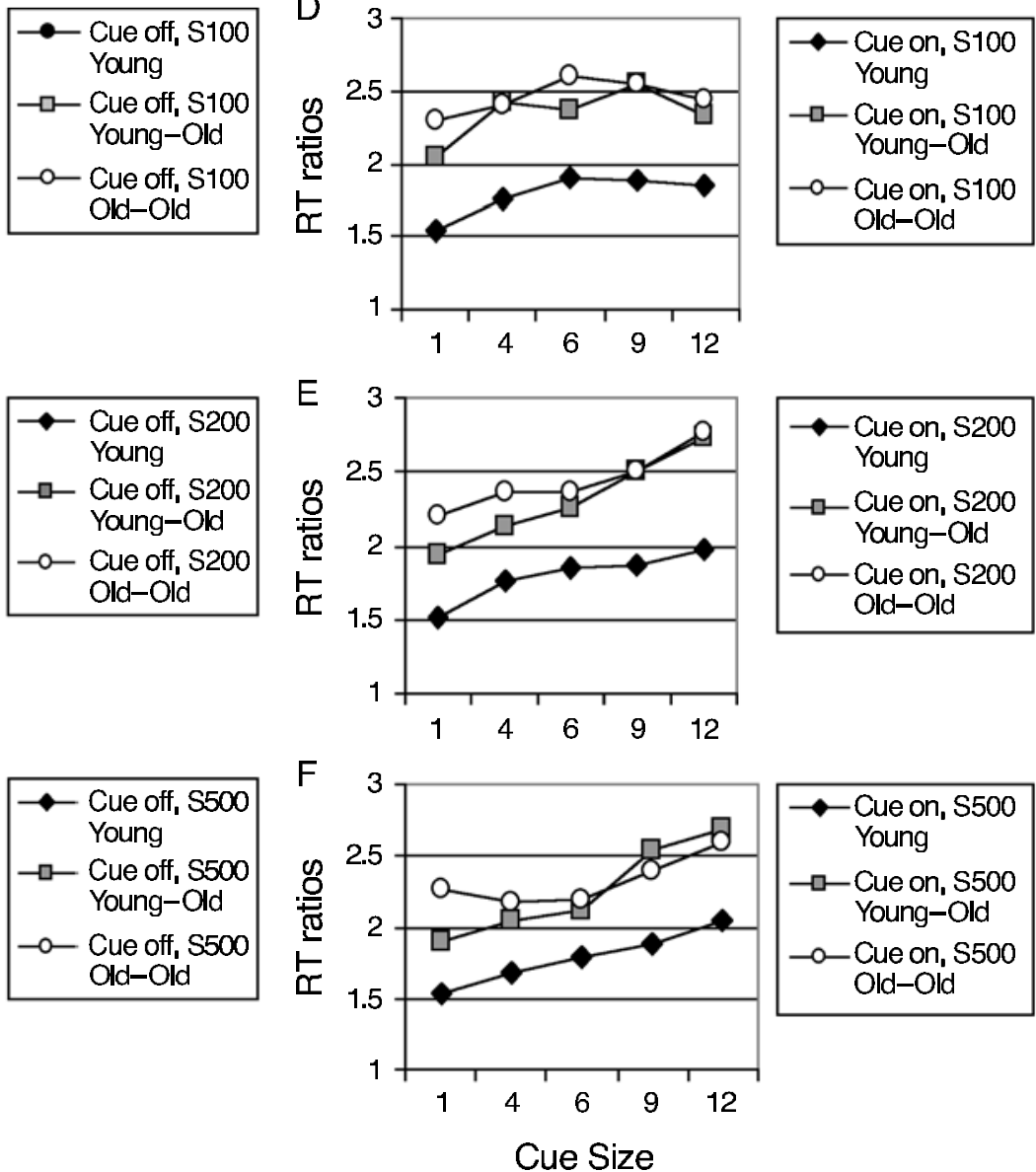

Cue Size

Figure 5. Experiment 2: conjunction search reaction time (RT) ratios plotted as a function of valid cue size and cue duration in young, young-old, and old-old groups. In panels A-C, the cue was turned off before the search array appeared: (A) cue-array stimulus onset asynchrony (SOA), $100 \mathrm{msec}$; (B) cue-array SOA, $200 \mathrm{msec}$; (C) cue-array SOA, $500 \mathrm{msec}$. In panels D-F, the cue remained on after the search array appeared: (D) cue-array SOA, $100 \mathrm{msec}$; (E) cue-array SOA, $200 \mathrm{msec}$; (F) cue-array SOA, 500 msec.

Scaling effects appeared to develop at about the same rate for feature as for conjunction search, suggesting a process common to both. This is in contrast to the view of Treisman and colleagues that they are separate processes (Treisman, 1985). We had previously reported that attentional scaling effects were not fully developed at short SOAs (Greenwood \& Parasuraman, 1999), but that was based on an informal comparison between experiments, rather than on the within-subjects design employed here.

The increase in cue size effect with time could be due to several factors. First, the scaling of the attentional focus may develop slowly, in contrast to shifting of the attentional focus. Second, attentional cuing effects, whether shifting or scaling, may develop more slowly within the more demanding search setting used here, as compared with the nonsearch settings in which their time course has typically been measured (Greenwood et al., 1993; Müller \& Rabbitt, 1989).

Cuing effects changed over the adult lifespan, increasing from the young to the young-old individuals but de- creasing in the old-old individuals. Differences between young-old and old-old individuals in attentional processing have been reported previously (Greenwood \& Parasuraman, 1994, 1999). A scatterplot of the relation between age and conjunction slope (Figure 6) shows that, after age 75 , a large number of individuals showed smaller slopes, as compared with slopes from those younger than 75 . This is consistent with a recent report showing slight agerelated change in cognition between 60 and 70 years, followed by marked change between 70 and 80 years (Rabbitt, Diggle, Smith, Holland, \& McInnes, 2001).

Examination of Figures 4 and 5 indicates that the oldold group showed a selectively reduced ability to scale the attentional focus to the smallest cues in both feature and conjunction searches. In contrast, their patterns of search speed following middle-sized or large cues were very similar to those of the young-old group. Age also appeared to slow the time course of attentional scaling. In conjunction search in the young group, search was facilitated by small cues only $100 \mathrm{msec}$ after cue onset. Such facilitation was 


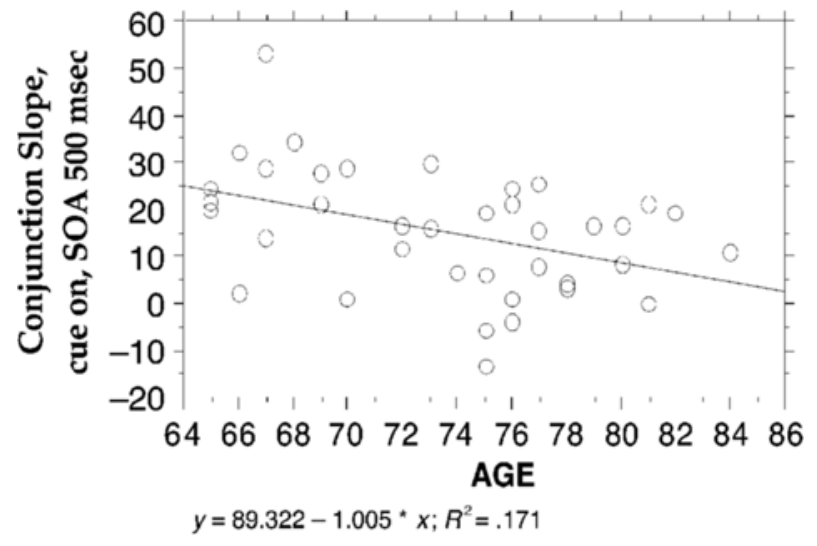

Figure 6. Experiment 2: scatterplot of conjunction search slopes of reaction-action/cue-size functions plotted as a function of age for all the older participants.

not evident in the young-old group until $200 \mathrm{msec}$ after cue onset. In the old-old group, modulation of search speed was also evident at $200 \mathrm{msec}$, but only when cues encompassed six or more letters. Search following an element-sized cue was not facilitated in this group even $500 \mathrm{msec}$ after cue onset. The results from feature search are similar. Madden and Gottlob (1997) also observed that the attentional focus broadened with SOA in a flanker task, but unlike in the present study, no age effect was seen. It can be concluded that whereas the time course of scaling is slowed with age, the effect of scaling is initially enhanced, then later reduced, so that the ability to either achieve or benefit from a constricted attentional focus becomes impaired in advanced aging.

\section{EXPERIMENT 3}

Our three-component model is concerned with the processing components involved in the detection of a target within a cluttered field. As was discussed in the introduction to this article, neurophysiological and neuroimaging work has indicated that attention exerts the strongest effects in the presence of distractors. Beginning with the work of Motter, a number of studies have shown that the firing rates of neurons in V1 and V4 are modulated by attention, but only when there are distractors near the receptive field (Gilbert, Ito, Kapadia, \& Westheimer, 2000; Motter, 1993, 1994). The importance of distractors in attentional modulation is implicitly acknowledged in the visual search paradigm, to which distractors are intrinsic. However, the effect of distractors on scaling visuospatial attention has been little investigated. With the exception of one group (Castiello \& Umiltà, 1990), attentional scaling has been studied only in the presence of distractors (Eriksen \& St. James, 1986; Greenwood \& Parasuraman, 1999; LaBerge et al., 1991; Luo, Greenwood, \& Parasuraman, 2001). In Experiment 3, we investigated attentional scaling in the absence of distractors.
Another important issue concerns the nature of attentional scaling. What information is conveyed by the size of a precue? In our example at the beginning of this article, it is assumed that the size of a disturbed region in a tree cues the observer to the size and, consequently, the identity of the actor hidden in the tree. However, it is not certain how the information conveyed by a size precue is used. Is it applied to the size of a region of space, to the size of an object, or to both? Previous work, our own included, has varied the size of the cued region, but not the size of the targets. Yet the size of an object, often known in advance, is an important cue to its identity. In order to address this question, in Experiment 3, we manipulated target size together with cue size.

\section{Method}

\section{Participants}

There were 15 young college student volunteers and 25 older community-dwelling volunteers. The older individuals were divided by age into two groups of 15 young-old (65-74 years of age) and 10 old-old (75-84 years of age). Demographic characteristics of these groups are presented in Table 1.

\section{Stimuli}

Figure 1C illustrates the stimuli and procedures. The cued discrimination task required a consonant/vowel decision about a letter target whose location and size were cued with varying validity. Each trial began with a fixation point for $500 \mathrm{msec}$, followed by an open square precue. Following a cue-target SOA of $500 \mathrm{msec}$, a target letter appeared for $2,000 \mathrm{msec}$. The task required a speeded response indicating whether the target was a consonant (50\% of the trials) or a vowel (50\% of the trials). Precues were small, medium, or large squares, each side measuring $1.9^{\circ}, 4.5^{\circ}$, and $8.5^{\circ}$ of visual angle, respectively. Targets were small, medium, or large letters, measuring $1.4^{\circ} \times 1.4^{\circ}, 3.8^{\circ} \times 3.5^{\circ}$, and $6.7^{\circ} \times 7.2^{\circ}$ of visual angle, respectively. Precues were valid for size and location on $60 \%$ of the trials (360 trials), invalid for size and location on $10 \%$ of the trials (30 trials at each combination of cue size and target size), valid for size but not location on $10 \%$ of the trials ( 30 trials at each combination of cue size and target size), and valid for location but not for size on $10 \%$ of the trials ( 30 trials at each combination of cue size and target size). Precues were neutral (a centered asterisk) on $10 \%$ of the trials (30 trials at each target size).

\section{Accuracy}

\section{Results}

Accuracy ranged from .909 for the young-old group when both the cue and the target were small (element sized) but invalidly cued for location to .990 for the old-old group when both the location and the size were invalidly cued.

\section{Reaction Time}

Each participant's RT was divided by the mean of pre- and postsession SRT medians to adjust for age-related slowing. An omnibus analysis of variance (ANOVA) was performed on these RT ratios. There was no main effect of age group and no interactions involving age group. There were significant main effects of validity $[F(1,37)=373.35, p<.0001]$, cue size $[F(2,74)=23.65, p<.0001]$, and target size 


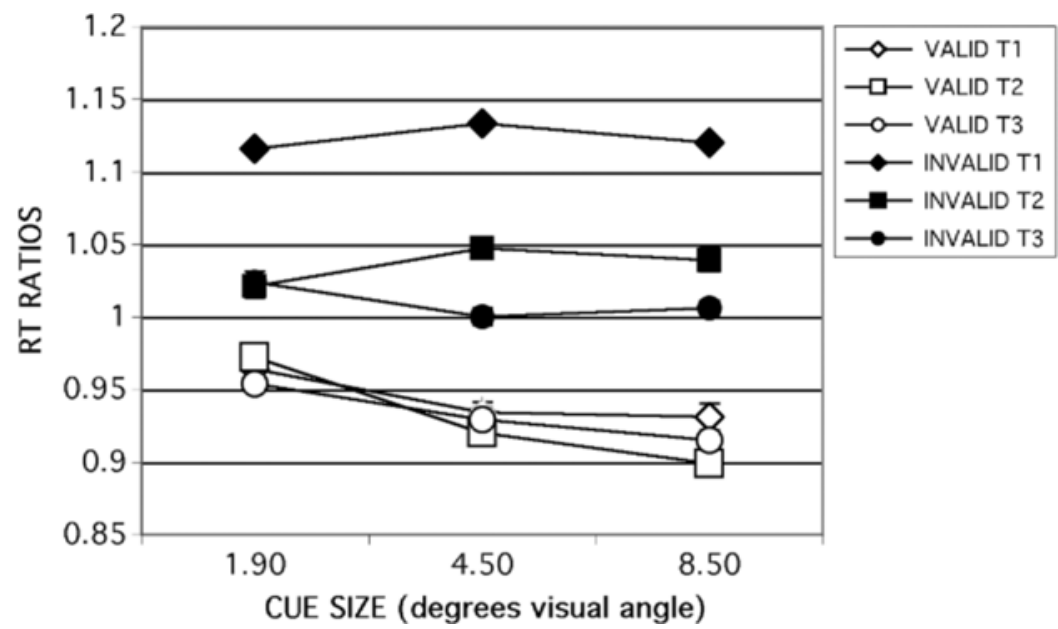

Figure 7. Experiment 3: discrimination reaction time (RT) ratios plotted as a function of cue validity and cue size.

$[F(2,74)=125.00, p<.0001]$ and a three-way interaction $[F(4,148)=6.72, p<.0001]$. Discrimination RT was faster for the two larger target sizes $[F(2,74)=3.68, p=.004]$ and decreased with cue size $[F(2,74)=55.89, p<.0001]$. These two factors interacted with each other $[F(4,148)=4.27$, $p=.003]$, but not with age group (Figure 7).

These results showing a decrease in RT with increasing cue size deviate not only from our previous manipulations of cue size in the presence of distractors, but also from the results of Castiello and Umiltà's (1990) manipulation of cue size in the absence of distractors. On the basis of this evidence, we hypothesized that the decrease in cue size observed in the present study was due to the unpredictability of target size. Therefore, we reran this task in different individuals, using a subset of trials in which the validity of both cue size and cue location varied, whereas target size was fixed at the smallest size. The stimuli were otherwise the same as those described above. Under these conditions, discrimination RT increased modestly with cue size $[F(2,26)=3.51, p<.05$; Figure 8$]$, in agreement with findings from previous studies that varied cue size with fixed target size in both the presence (Greenwood \& Parasuraman, 1999; Greenwood et al., 1997) and the absence (Castiello \& Umiltà, 1990) of distractors. Moreover, the size of the change in RT with cue size in the absence of distractors (about $20 \mathrm{msec}$ ) was comparable to that observed by Castiello and Umiltà under similar conditions. As in the full task, described above, there was no effect of age.

\section{Discussion}

These results emphasize that attentional scaling is optimized for task demands. Under conditions in which the target was unpredictable with regard to both location and size, the optimal attentional scale was broad. In contrast, under conditions in which target size, although not target location, was predictable, the optimal attentional scale was constricted around element size. Considered together, evidence from all three experiments indicates that the focus of attention becomes set to an "experiment-wise" scale that is optimal for such task demands as target-distractor similarity, array size, and element size. Presentation of cues prior to the search array dynamically alters that scale, thereby speeding or slowing search, depending on the compatibility between precue information and demands of the search array.

\section{GENERAL DISCUSSION}

In this work, we sought evidence in support of our model of visual search. This involved both confirming previous findings on modulation of visual search speed by

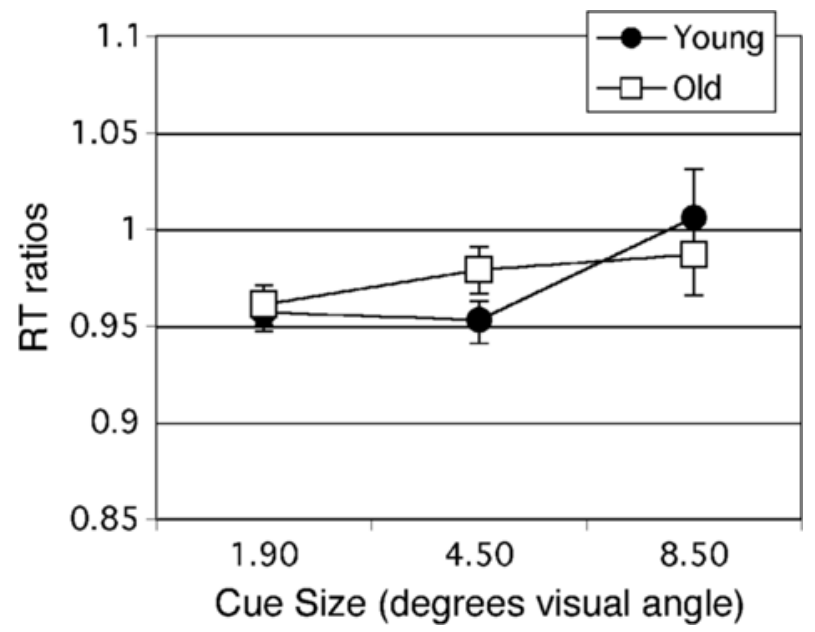

Figure 8. Experiment 3: discrimination reaction time (RT) ratios plotted as a function of cue validity and cue size. 
precue precision and extending those findings to show that attentional scaling is a dynamic, flexible, and automatic processing component of visuospatial attention. The following evidence was obtained. (1) Visual search is most strongly facilitated when precues are validly located and close in size to array elements. These conditions allow the attentional focus to be constricted around the target (Experiment 1). (2) However, this benefit applies only when target size is fixed. When target size is unpredictable, the optimal scale for search is larger, encompassing multiple array elements (Experiment 3). (3) The optimal scale is also larger when cues inaccurately indicate target location (Experiment 1). (4) Moreover, this is true whether distractors are present (Experiment 1) or absent (Experiment 3). (5) In the absence of distractors, attentional scaling is weaker but is otherwise deployed as it is in the presence of distractors (Experiment 3). (6) Attentional scaling to precues appears to be obligatory-that is, not strategic. This is based on evidence that attentional scaling has a time course and is unaffected by precue duration (Experiment 2). Moreover, an element-sized precue does not necessarily speed discrimination (Experiment 3 ).

Considered as a whole, this evidence suggests the existence of a robust, nonstrategic mechanism that uses prior information to scale the attentionalfocus in preparation for search demands. But what is the entity around which the attentional focus is scaled? Is it the region of visual space within which the target is expected to appear, or is it the expected size of the target? The answer appears to depend on the context in which the cuing information was imparted. In Experiments 1 and 2, in which target size was fixed and known in advance-typical in most of the visual search literature - the participants appeared to rely on their prior knowledge of target size so that the optimal scale for search was based on element size. In Experiment 3 when target size was unpredictable, the participants processed targets most rapidly following a large cue. When target size was made predictable, the optimal scale was again element sized. This suggests that when there is uncertainty, the optimal scale is large and diffuse. Thus, in visual search, the focus of attention is scaled in accordance with overall task demands, rather than only to the size of array elements or the size of the relevant region. In the real world, such an attentional scaling mechanism could allocate limited processing resources efficiently by deploying them in a concentrated or a diffuse manner according to task demands within the region of space in which events are most likely to appear.

These results are consistent with our model of visual search, in which, on a given search trial and in response to prior information, visuospatial attention is initially scaled and then shifted in advance of a possible eye movement toward those elements possessing target properties. We acknowledge that other conceptualizations of visual search might be able to account for our data. Although aspects of our results are consistent with both FIT and GS models, those models would need modification in order to account for evidence of attentional scaling.
Attentional scaling appears to be particularly important with advancing age, exerting the greatest effect on search performance of young-old individuals. After 75 years of age, however, a selective impairment develops in the ability to achieve or maintain a small, element-sized, attentional focus. Overall, then, aging alters the ability to deploy visuospatial attention, at first slowing the course of its development and increasing its dependence on external cues, but eventually impairing its ability to focus on a small region of space. These age-related changes may underlie the relative impairment of older people in performing search for conjunctions of features (Plude \& DoussardRoosevelt, 1989) or under conditions of what is termed "hard" search (Duncan \& Humphreys, 1989) when there is high target-distractor similarity (Scialfa, Esau, \& Joffe, 1998).

\section{Effects of Precue Size on Speed of Popout}

Contrary to Treisman's view that feature search is preattentive (Treisman \& Gelade, 1980), precue size modulated speed of popout in a fairly linear manner. The RT benefit of an element-sized precue on popout developed rapidly with time from cue onset. Figure 4 shows that in the young group, the benefit of a small cue was evident $100 \mathrm{msec}$ after cue onset, whereas the cost of a larger cue was not. Thus, in the young group, the attentional focus could be constricted faster than it could be expanded. This time course was slower in the older groups. In young-old individuals, small cues did not affect popout until $200 \mathrm{msec}$ after cue onset (Figure 4). This was true whether the cue remained on during the array (Figures 4D-4F) or was turned off before the array appeared (Figures 4A-4C). The presence of distractors appears to strengthen the effects of attentional scaling, but not to change their nature. Although not, strictly speaking, a popout condition, the search task without distractors in Experiment 3 induced RT facilitation with small, relative to large, precues. However, this was seen only when target size was unvarying. Under this condition of search without distractors, age had no effect. This suggests that the age effects seen in more typical search tasks may be related to the need to filter or otherwise process distractors.

However, deployment of a constricted attentional focus is not always advantageous. Search is slowed following a small cue that does not enclose the target (Figures 3A and 3B) and when target size is unpredictable (Figure 7). Under those conditions, search is fastest following a large cue. It may be that when the target appears at a distance from the center of the attentional focus, a more diffuse focus with a shallow gradient can better detect a popout target. Evidence from Experiment 2 suggests that constriction of the attentional focus occurs faster than expansion. Thus, it may be that when target size is unpredictable, setting the attentional focus to a large scale facilitates detection of peripheral targets. Under such conditions, detection of a small target would be speeded by the ability to rapidly constrict the attentional focus. 
The advantage of precue precision on popout appears to be reduced in old age. In young individuals, the optimal scale was modeled on element size, whereas in both youngold and old-old individuals the optimal scale encompassed several array elements. This effect developed over time (SOA) and was most marked in old-old individuals (Figure 4). In the real world, having a larger attentional focus could allow older individuals to scan more widely for a popout target. A broadened focus could also facilitate contrast and, hence, discrimination between the target and the surrounding distractors. Furthermore, in the oldold group, there was actually a cost from very precise precues that, under some conditions, equaled the cost of the largest precue (Figure 4F). This cost may derive from an inability of old-old individuals to obtain a processing benefit from constriction of the attentional focus. Finally, in the absence of distractors, effects of age on scaling were not seen (Experiment 3), perhaps because scaling effects are smaller when distractors are not present.

\section{Effects of Precue Size on Speed of Conjunction Search}

Attentional scaling was deployed differently when the target of search was a conjunction of features. In contrast to the case with popout targets, which older participants detected best following larger cues, in both young-old and young participants conjunction search was facilitated by small (element-sized) valid cues. This is consistent with our previous findings (Greenwood \& Parasuraman, 1999; Greenwood et al., 1997) that greater precision of prior information about the region of space where a target is likely to occur facilitates discrimination of conjunction targets within that region. Presumably, this occurs through dynamic adjustment of the attentional focus around the cued region. Old-old individuals responded differently. Although they also exhibited effects of scaling on conjunction search, the effects were not linear at any SOA. Examination of Figure 5 indicates that smaller precues often slowed search in old-old individuals, in contrast to the facilitatory effect of such precues in young and young-old individuals.

Thus, as aging advances, there are changes in the way visuospatialattention is deployed. Initially, between the ages of 65 and 74 years, there is increased dependence on precue size information. Whereas the benefits conferred by small cues to target location on conjunction search in the young-old group were similar to those experienced by the young group, the costs of larger cues were greater in the young-old group (Figure 5). After an age of 75 years, the benefit of small cues was reduced. Moreover, all the older participants were slower than the young adults to scale the attentional focus (Experiment 2, Figure 5). This finding may be related to one seen previously in nonsearch discrimination tasks in which disengagement of visuospatial attention away from an invalidly cued location is slowed, but only in old-old individuals (Greenwood \& Parasuraman, 1994).
There were also marked age differences in responding when cues were invalidly located. In young participants, conjunction search was dramatically slowed when the cues were small and invalidly located. Such cues produced costs that were both greater than the costs from larger invalid cues and greater than the costs from invalid cues in older individuals (Experiment 1, Figure 3A).

That young-old individuals experience stronger effects of area cuing than do young individuals has been reported previously. Hartley et al. (1992) used a "flanker" task (LaBerge \& Brown, 1989) in which horizontally arranged words acted as a "cue" to set the scale of the attentional focus for the response to a subsequent probe stimulus within the cued region. RT slowed with increasing distance from the center of the cue, producing the V-shaped RT function reported by LaBerge and Brown. Although the young-old participants (mean age, 72 years) of Hartley et al. had a consistently steeper slope of RT/eccentricity function than did young participants across the three experiments, the differences were not significant in every case. Gottlob and Madden (1999) also observed that their young-old adults (mean age, 68 years) had steeper slopes than did young adults in a flanker task. Thus, the present findings that cue-size/RT functions were steeper in the young-old group than in the young group are consistent with previous findings (Gottlob \& Madden, 1999; Hartley et al., 1992). However, whereas Gottlob and Madden interpreted their results as indicating a more "constricted" attentional focus in the young-old individuals, we interpret our results as indicating that the young-old individuals scale their attentional focus more closely to cue characteristics. This interpretation is consistent with an earlier conclusion that older people are more reliant than young people on location cues in a memory search task (Madden, Pierce, \& Allen, 1992).

\section{Underpinnings of the Age-Related Change in Deployment of Attentional Scaling}

What underlies this age-related change in the modulation of search speed by attentional scaling? Increased dependence of young-old individuals on prior information about a target event may arise from a reduced ability to employ bottom-up mechanisms to discriminate the target from the distractors in the course of search. By this view, difficulty in discriminating the target would lead to a compensatory increase in dependence on attentional scaling in order to isolate the target from surrounding distractors. Such increased dependence on attentional processes in the young-old individuals was not evident in the old-old ones. The old-old group appeared to preferentially deploy a chronically broadened attentional focus in search, so that search proceeded most efficiently when the precue encompassed multiple array elements. This may reflect either (1) an inability to scale the attentional focus to an element-sized cue or (2) an inability to benefit from such scaling. The latter might occur if inefficient bottom-up mechanisms result in a need to compare target and distractors within the attentional focus in order to discriminate them. 
That young individuals derive a smaller RT benefit from valid cues overall than do young-old individuals (Figure 2B) suggests they may automatically scale the attentional focus to the size of array elements. This could be done as a task-wise "default" setting under bottom-up control arising from their experience with the unchanging size of array elements. There is evidence that a small attentional focus is more "concentrated" and less susceptible to interference from nearby distractors than is a larger attentional focus (Eriksen \& St. James, 1986). If so, in young people, a valid element-sized cue would provide relatively little additional benefit, because attention would already have been scaled to element size by bottom-up processes.

On the other hand, such a concentrated attentional focus may be a disadvantage if the cues turn out to be invalid and the target must be sought outside the cued region. Reduced effectiveness of events outside the cued region in attracting attention could slow detection of a target that lies outside cue borders and, presumably, either at or beyond the "fringe" of the attentional focus first postulated by William James (1890/1981). We reported previously that when cues are invalidly located, search is fastest when the cue is large enough to encompass several array elements (Greenwood, Alexander, \& Parasuraman, 1999; Greenwood et al., 1997). Therefore, an element-sized attentional focus could generate a cost if located away from the target, due to the added requirement to disengage the attentional focus away from the cued location (Greenwood \& Parasuraman, 1994; Posner, 1980; Posner, Walker, Friderich, \& Rafal, 1984). This was seen in Experiment 1, in which young, but not old, participants were particularly slowed when the cue was small but invalidly located. That young individuals experience greater costs than do older individuals from small invalid cues suggests further that the young people deploy an attentional focus that is more resistant to distraction. Thus, in young people, having visuospatial attention constricted around the cued region appears to impede the ability to detect a target located outside the cued region. This stands in contrast to the older people, who showed smaller costs than did young people following an element-sized cue (Experiment 1, Figures 2 and 3). This suggests that older individuals deploy a more diffuse, less concentrated attentional focus, which is, therefore, more subject to distraction, including interference from nearby array elements, including the target.

These findings lead to the view that young individuals deploy an attentional focus that is (1) more dependent on bottom-up endogenous control and less dependent on exogenous attentional control and (2) more concentrated and, therefore, less susceptible to interference from distractors. By contrast, the attentionalfocus in young-old individuals may be under more exogenous control than is that of the young and, thereby, more subject to modulation by precues. The attentional focus of old people may have a larger default size that is also more diffuse and, therefore, more susceptible to distraction than is that of young people. Finally, in old-old people, there is a failure of the ability to constrict the attentional focus. Old-old individuals appear to be selectively impaired in the ability to attain or benefit from an attentional focus scaled to element size.

\section{Physiological Evidence of Attentional Scaling}

Suggesting a physiological basis for attentional scaling is single-cell evidence showing that spatial cuing results in the apparent shrinkage of receptive fields around a target, excluding a distractor (Luck \& Hillyard, 1994; Moran \& Desimone, 1985). Other studies have reported attentional modulation of single-cell firing, with the suppression of firing from the presence of distractors being reduced by the deployment of focal attention (Motter, 1993). Gilbert and colleagues recently reported that in both human and infrahuman primates, large precues induced a greater increase in perceived brightness of a line target than did small cues. This result suggests a physiological basis for attentional scaling effects (Gilbert et al., 2000).

The influential biased competition model of visual processing (Desimone \& Duncan, 1995) argues that prior knowledge of target spatial location exerts top-down control, which can bias processing toward the target. In these terms, greater precision of such prior information would increase the bias in favor of the target, as opposed to distractors. The shrinking of receptive fields around a target subsequent to spatial cuing would effectively exclude nearby distractors (Luck \& Hillyard, 1994; Moran \& Desimone, 1985).

Desimone and Duncan (1995) argued that the source of the top-down selection of spatial location arises in the posterior parietal and/or the prefrontal cortex. Others have found evidence of mediation of visuospatial attention in parietal regions (Corbetta, 1998; Davidson \& Marrocco, 2000). Although there is evidence for a role for frontal (near the frontal eye fields) as well as parietal (intraparietal sulcus) regions in shifting visuospatial attention (Nobre et al., 1997), scaling of visuospatial attention may be mediated in more inferior brain regions. The temporal-parietal cortex was shown to be selectively active during a global/ local task that required repeated attentional scaling (Fink et al., 1996, 1997; Yamaguchi, Yamagata, \& Kobayashi, 2000). This points to a role for this region in top-down scaling of attention at a spatial location.

To the extent that popout is a bottom-up process, presumably it could develop from biased competition by virtue of the salience of the target alone. Size and location precues, such as those used in the present designs, could create top-down control over such competition, giving an advantage to items appearing within the cued region. Bottomup detection of the target amid distractors could, therefore, be modulated by the top-down processes triggered and guided by size and location precues. The observed speeding of popout by precise precues could arise if the effect of scaling is to limit the number of items between which competition occurs. From this point of view, it appears evident that some interaction must occur between bottom-up and top-down control of search processes.

There may be an age-related difference in deployment of top-down and bottom-up processes. As was suggested 
above, young individuals appear to scale their attentional focus to the size of array elements. This scaling is presumably driven by bottom-up processing. In an experimental task, this could occur during practice or the first few test trials. Ito and Gilbert (1999) have shown, in psychophysical studies in both humans and monkeys, that effects of distributed or focal location cues change over the course of training. Age-related declines in central visual processing could lead to reduced efficiency in bottom-up processes of target discrimination. Consequently, the role of top-down mechanisms could increase, producing the heightened effects of precue size seen in young-old individuals. Also, deployment of a broader attentional focus in aging could enhance comparisons between the target and the surrounding distractor elements and, thus, allow older people to perform as accurately as young people. Evidence from imaging work indicates that when older adults perform tasks at an accuracy level similar to that for young adults, they activate additional brain regions, interpreted as reflecting compensation (Cabeza, 2002; Grady, 1998). The age-related changes in visuospatial attention in visual search described in the present study could be the behavioral reflection of such a compensatory process.

The reduction in benefits of small precues in old-old individuals in Experiment 2 may be due to further declines in the efficiency of visual processing with advancing age. The reduction could also be due to an age-related reduction in the efficiency of attentional mechanisms in the striate and extrastriate cortex (Gilbert et al., 2000; Ito \& Gilbert, 1999; Motter, 1993, 1994). The evidence that agerelated decline of cortical integrity is confined to any one region is weak and conflicting (Greenwood, 2000). However, there is evidence that aging is accompanied by hypometabolism of prefrontal, parietal, and temporal association regions (Jernigan et al., 1991; Martin, Friston, Colebatch, \& Frackowiak, 1991; Smith et al., 1999). Such an explanation is consistent with a recent report of reduced effects of precue size on search in individuals at increased risk of Alzheimer's disease by virtue of possession of the $\varepsilon 4$ allele of the apolipoprotein gene (Greenwood, Sunderland, Friz, \& Parasuraman, 2000). Individuals who are $\varepsilon 4$ carriers have been shown to exhibit hypometobolism in the prefrontal, parietal, and temporal association cortex in middle age (Reiman et al., 1996).

Taking a different approach, it has been argued that detection of features and conjunctions of features occurs in parallel (McElree \& Carrasco, 1999), contrary to the views of Treisman and Gelade (1980) and of Cave and Wolfe (1990) that only conjunction search is serial and contrary to the claims of Duncan and Humphreys (1989) that all search is serial. In this view, the kind of set size effects taken as evidence for serial deployment of covert attention could also arise from capacity limits in parallel processing (Townsend $\&$ Ashby, 1984) or from reductions in discriminability due to increased noise in the decision process (Kinchla, Chen, \& Evert, 1995; Shaw, 1984). Applying such interpretations to the present study, cue size effects might have arisen from modulation of the noise in the decision process by changing the effective set size. Even with this interpretation, however, some mechanism would be necessary for the process of attentional scaling itself.

\section{REFERENCES}

Braun, J., \& SAGI, D. (1991). Texture-based tasks are little affected by second tasks requiring peripheral or central attentive fixation. Perception, 20, 483-500.

CABEZA, R. (2002). Hemispheric asymmetry reduction in older adults: the HAROLD model. Psychology \& Aging, 17, 85-100.

Caggiano, D., Greenwood, P. M., \& Parasuraman, R. (2001, April). Aging alters the role of saccades in attentional scaling effects on visual search. Paper presented at the meeting of the Cognitive Aging Society, Atlanta.

Carter, J. E., Obler, L., Woodward, S., \& Albert, M. L. (1983). The effect of increasing age on the latency for saccadic eye movements. Journals of Gerontology, 38, 318-320.

Castiello, U., \& Umiltà, C. (1990). Size of the attentional focus and efficiency of processing. Acta Psychologica, 73, 195-209.

CAVE, K. R., \& BICHOT, N. P. (1999). Visuospatial attention: Beyond a spotlight model. Psychonomic Bulletin \& Review, 6, 204-223.

CAVE, K. R., \& WolfE, J. M. (1990). Modeling the role of parallel processing in visual search. Cognitive Psychology, 22, 225-271.

CORBE TTA, M. (1998). Frontoparietal cortical networks for directing attention and the eye to visual locations: Identical, independent, or overlapping neural systems? Proceedings of the NationalAcademy of Sciences, 95, 831-838.

DAVIDSON, M. C., \& MARrocco, R. T. (2000). Local infusion of scopolamine into intraparietal cortex slows covert orienting in rhesus monkeys. Journal of Neurophysiology, 83, 1536-1549.

DESIMONE, R., \& DunCAN, J. (1995). Neural mechanisms of selective visual attention. Annual Review of Neuroscience, 18, 192-222.

DiAmond, M. R., Ross, J., \& Morrone, M. C. (2000). Extraretinal control of saccadic suppression. Journal of Neuroscience, 20, 3449-3455.

DunCAN, J., \& Humphreys, G. W. (1989). Visual search and stimulus similarity. Psychological Review, 96, 433-458.

ERIKSEN, C. W., \& ST. JAMES, J. D. (1986). Visual attention within and around the field of focal attention: A zoom lens model. Perception \& Psychophysics, 40, 225-240.

FAust, M. E., \& BALOTA, D. A. (1997). Inhibition of return and visuospatial attention in healthy older adults and individuals with dementia of the Alzheimer type. Neuropsychology, 11, 13-29.

Fink, G. R., Halligan, P. W., Marshall, J. C., Frith, C. D., FrackOWIAK, R. S., \& DOLAN, R. J. (1996). Where in the brain does visual attention select the forest and the trees? Nature, 382, 626-628.

Fink, G. R., Halligan, P. W., Marshall, J. C., Frith, C. D., FrackOWIAK, R. S., \& DOLAN, R. J. (1997). Neural mechanisms involved in the processing of global and local aspects of hierarchically organized visual stimuli. Brain, 120(Pt. 10), 1779-1791.

Gilbert, C., Ito, M., Kapadia, M., \& Westheimer, G. (2000). Interactions between attention, context and learning in primary visual cortex. Vision Research, 40, 1217-1226.

Gilchrist, I. D., Heywood, C. A., \& Findlay, J. M. (1999). Saccade selection in visual search: Evidence for spatial frequency specific betweenitem interactions. Vision Research, 39, 1373-1383.

GotTlob, L. R., \& Madden, D. J. (1999). Age differences in the strategic allocation of visual attention. Journals of Gerontology, 54, 165-172.

GRADY, C. L. (1998). Brain imaging and age-related changes in cognition. Experimental Gerontology, 33, 661-673.

GREENWOOD, P. M. (2000). The frontal aging hypothesis evaluated. Journal of the International Neuropsychological Society, 6, 705-726. Greenwood, P. M., Alexander, G. E., \& Parasuraman, R. (1999, Month). Progression in early Alzheimer disease increases costs but not benefits of cue validity in visuospatial attention. Paper presented at the meeting of the Society for Neuroscience, Miami, FL.

Greenwood, P. M., \& Parasuraman, R. (1994). Attentional disengagement deficit in nondemented elderly over 75 years of age. Aging \& Cognition, 1, 188-202.

Greenwood, P. M., \& PARASUraman, R. (1999). Scale of attentional focus in visual search. Perception \& Psychophysics, 61, 837-859. Greenwood, P. M., Parasuraman, R., \& Alexander, G. E. (1997). 
Controlling the focus of spatial attention during visual search: Effects of advanced aging and Alzheimer disease. Neuropsychology, 11, 3-12.

Greenwood, P. M., PArasuraman, R., \& Haxby, J. (1993). Changes in visuospatial attention over the adult lifespan. Neuropsychologia, 31, 471-485.

Greenwood, P. M., Sunderland, T., Friz, J. L., \& Parasuraman, R. (2000). Genetics and visual attention: Selective deficits in healthy adult carriers of the varepsilon 4 allele of the apolipoprotein $\mathrm{E}$ gene. Proceedings of the National Academy of Sciences, 97, 11661-11666.

Hartley, A. A., Kieley, J., \& MCKenZIE, C. R. M. (1992). Allocation of visual attention in younger and older adults. Perception \& Psychophysics, 52, 175-185.

Hawkins, H. L., Hillyard, S. A., Luck, S. J., Mouloua, M., DownING, C. J., \& WoODWARD, D. P. (1990). Visual attention modulates signal detectability. Journal of Experimental Psychology, 16, 802-811.

INTRILIGATOR, J., \& CAVANAGH, P. (2001). The spatial resolution of visual attention. Cognitive Psychology, 43, 171-216.

ITO, M., \& Gilbert, C. D. (1999). Attention modulates contextual influences in the primary visual cortex of alert monkeys. Neuron, 22, 593-604.

JAMES, W. (1981). The principles of psychology (Vol. 1). Cambridge, MA: Harvard University Press. (Original work published 1890)

Jernigan, T. L., Archibald, S. L., Berhow, M. T., Sowell, E. R., FosTER, D. S., \& HESSELINK, J. R. (1991). Cerebral structure on MRI: Pt. I. Localization of age-related changes. Biological Psychiatry, 29, 55-67.

Kinchla, R. A., Chen, Z., \& Evert, D. L. (1995). Precue effects in visual search: Data or resource limited? Perception \& Psychophysics, 57, 441-450.

LABERGE, D., \& BROWN, V. (1989). Theory of attentional operations in shape identification. Psychological Review, 96, 101-124.

LaBerge, D., Brown, V., Carter, M., Bash, D., \& Hartley, A. (1991). Reducing the effects of adjacent distractors by narrowing attention. Journal of Experimental Psychology: Human Perception \& Performance, 17, 65-76.

LaBerge, D., Carlson, R. L., Williams, J. K., \& Bunney, B. G. (1997). Shifting attention in visual space: Tests of moving-spotlight models versus an activity-distribution model. Journal of Experimental Psychology: Human Perception \& Performance, 23, 1380-1392.

LuCK, S. J., \& Hillyard, S. A. (1994). Spatial filtering during visual search: Evidence from human electrophysiology. Journal of Experimental Psychology: Human Perception \& Performance, 20, 1000-1014

Luo, Y. J., Greenwood, P. M., \& PARAsuraman, R. (2001). Dynamics of the spatial scale of visual attention revealed by brain event-related potentials. Cognitive Brain Research, 12, 371-381.

Madden, D. J., \& GotTlob, L. R. (1997). Adult age differences in strategic and dynamic components of focusing visual attiention. Aging, Neuropsychology, \& Cognition, 4, 185-210.

Madden, D. J., Pierce, T. W., \& Allen, P. A. (1992). Adult age differences in attentional allocation during memory search. Psychology \& Aging, 7, 594-601.

Martin, A. J., Friston, K. J., Colebatch, J. G., \& Frackowiak, R. S. J. (1991). Decreases in regional cerebral blood flow with normal aging. Journal of Cerebral Blood Flow \& Metabolism, 11, 684-689.

Matin, E. (1974). Saccadic suppression: A review and an analysis. Psychological Bulletin, 81, 899-917.

MCElree, B., \& CARrasco, M. (1999). The temporal dynamics of visual search: Evidence for parallel processing in feature and conjunction searches. Journal of Experimental Psychology: Human Perception \& Performance, 25, 1517-1539.

MORAN, J., \& DESIMONE, R. (1985). Selective attention gates visual processing in the extrastriate cortex. Science, 229, 782-784.

MotTER, B. C. (1993). Focal attention produces spatially selective processing in visual cortical areas V1, V2, and V4 in the presence of competing stimuli. Journal of Neurophysiology, 70, 909-919.

MotTER, B. C. (1994). Neural correlates of feature selective memory and pop-outin extrastriate area V4. Journal of Neuroscience, 14, 2190-2199.

Motter, B. C., \& BelKy, E. J. (1998). The zone of focal attention during active visual search. Vision Research, 38, 1007-1022.

MüLleR, H. J., \& RABBITT, P. M. A. (1989). Reflexive and voluntary orienting of visual attention: Time course of activation and resistance to interruption. Journal of Experimental Psychology: Human Perception \& Performance, 15, 315-330.
Nakayama, K., \& MaKeben, M. (1989). Sustained and transient components of focal visual attention. Vision Research, 29, 1631-1647.

Nobre, A. C., Sebestyen, G. N., Gitelman, D. R., Mesulam, M. M., FRACKOWIAK, R. S., \& FRITH, C. D. (1997). Functional localization of the system for visuospatial attention using positron emission tomography. Brain, 120, 515-533.

Noesselt, T., Hillyard, S. A., WoldorfF, M. G., Schoenfeld, A., Hagner, T., Jancke, L., Tempelmann, C., Hinrichs, H., \& Heinze, H. J. (2002). Delayed striate cortical activation during spatial attention. Neuron, 35, 575-587.

Plude, D. J., \& Doussard-Roosevelt, J. A. (1989). Aging, selective attention and feature integration. Psychology \& Aging, 4, 98-105.

POSNER, M. I. (1980). Orienting of attention. Quarterly Journal of Experimental Psychology, 32, 3-25.

Posner, M. I., WAlker, J. A., Friderich, F. J., \& RAFAL, R. D. (1984). Effects of parietal injury on covert orienting of attention. Journal of Neuroscience, 4, 1863-1874.

Pre VIC, F. H. (1995, April). Oculomotor behavior during visual search in humans. Paper presented at the meeting of Society for Neuroscience, San Diego.

PrinZmetal, W., Presti, D. E., \& Posner, M. I. (1986). Does attention affect visual feature integration? Journal of Experimental Psychology: Human Perception \& Performance, 12, 361-369.

Rabbitt, P., Diggle, P., Smith, D., Holland, F., \& Mcinnes, L. (2001). Identifying and separating the effects of practice and of cognitive ageing during a large longitudinal study of elderly community residents. Neuropsychologia, 39, 532-543.

Reiman, E. M., Caselli, R. J., Yun, L. S., Chen, K., Bandy, D., Minoshima, S., Thibodeau, S. N., \& Osborne, D. (1996). Preclinical evidence of Alzheimer's disease in persons homozygous for the epsilon 4 allele for apolipoprotein E. New England Journal of Medicine, 334, $752-758$.

SCIALFA, C. T., ESAU, S. P., \& JOFFE, K. M. (1998). Age, target-distractor similarity, and visual search. Experimental Aging Research, 24, 337-358.

SHAW, M. L. (1984). Division of attention among spatial locations: A fundamental difference between detection of letters and detection of luminance increments. In H. Bouma \& D. G. Bouwhuis (Eds.), Attention and performance $X$ (pp. 109-120). Hillsdale, NJ: Erlbaum.

Smith, C. D., ANdersen, A. H., Kryscio, R. J., Schmitt, F. A., Kindy, M. S., Blonder, L. X., \& Avison, M. J. (1999). Altered brain activation in cognitively intact individuals at high risk for Alzheimer's disease. Neurology, 53, 1391-1396.

Theeuwes, J., Kramer, A. F., \& Atchley, P. (1999). Attentional effects on preattentive vision: Spatial precues affect the detection of simple features. Journal of Experimental Psychology: Human Perception \& Performance, 25, 341-347.

TownSEnd, J. T., \& AshBy, F. G. (1984). Stochastic modeling of elementary psychological processing. New York: Cambridge University Press.

Treisman, A. M. (1985). Preattentive processing in vision. Computer Vision, Graphics \& Imaging Processing, 31, 156-177.

Treisman, A. [M.] (1996). The binding problem. Current Opinion in Neurobiology, 6, 171-178.

Treisman, A. [M.], \& Gelade, G. (1980). A feature-integration theory of attention. Cognitive Psychology, 12, 97-136.

TREISMAN, A. [M.], \& GoRMICAN, S. (1988). Feature analysis in early vision: Evidence from search asymmetries. Psychological Review, 95, 15-48.

Treisman, A. [M.], \& SATO, S. (1990). Conjunction search revisited. Journal of Experimental Psychology: Human Perception \& Performance, 16, 459-478.

Yamaguchi, S., Yamagata, S., \& Kobayashi, S. (2000). Cerebral asymmetry of the "top-down" allocation of attention to global and local features. Journal of Neuroscience, 20, RC72.

Zelinsky, G. J., Rao, R. P. N., Hayhoe, M. M., \& Ballard, D. H. (1997). Eye movements reveal the spatiotemporal dynamics of visual search. Psychological Sciences, 8, 448-453.

ZELINSKY, G. J., \& SheINBerG, D. L. (1997). Eye movements during parallel-serial visual search. Journal of Experimental Psychology: Human Perception \& Performance, 23, 244-262.

(Manuscript received March 21, 2002; revision accepted for publication May 5, 2003.) 